\title{
密度分层流体中不同潜深拖曳回转体激发内波特性实验
}

\author{
王宏伟 ${ }^{1,2}$, 陈科 ${ }^{1,2^{*}}$, 尤云祥 ${ }^{1,2}$, 张新曙 1,2 \\ 1. 上海交通大学海洋工程国家重点实验室, 上海 200240; \\ 2. 高新船舶与深海开发装备协同创新中心, 上海 200240 \\ *联系人, E-mail: raulphan@sjtu.edu.cn
}

2016-08-08 收稿, 2016-10-28 修回, 2016-10-31 接受, 2017-01-11 网络版发表

国家自然科学基金(11072153, 11372184)资助

\begin{abstract}
摘要在具有强密度跃层的分层流体中, 采用沿水槽中纵剖面对称布置的电导率探头阵列方法, 对长径比为 7.7 的轴对称回转体在 5 种不同潜深下拖曳运动激发内波时空特性进行了系列实验. 结果表明: 存在一个与潜深无关 的临界转掞Froude数 $F r_{\mathrm{c}}=3.64$, 当Froude数 $F r<F r_{\mathrm{c}}$ 时, 在不同潜深下, 内波相关速度均与物体运动速度一致, 体积 效应内波为主控内波，但内波反对称度随 $F r$ 增大而增大，表明尾迹效应内波的影响也随着增大；同时，存在一个 内波峰值Froude数 $F r_{\mathrm{p}} \approx 1.33$, 当 $F r<F r_{\mathrm{p}}$ 时, 无量纲内波最大峰-峰幅值随 $F r$ 增大而增大, 而当 $F r>F r_{\mathrm{p}}$ 时, 无量纲内 波最大峰一峰幅值随 $F r$ 增大而减小。当 $F r>F r$ 时，在不同潜深下，内波相关速度均小于模型运动速度，其相关速度 Froude数 $F r_{\mathrm{iw}}$ 均在 0.7 1.4之间的一个条带内变化, 内波反对称度近似在某个小于 0.5 的常数附近波动, 尾迹效应内 波中对称部分略为占优，而且尾迹效应内波中对称和反对称部分的水平张角均近似为 $20.6^{\circ}$; 同时，无量纲内波最 大峰一峰幅值均随 $F r$ 增大而近似线性增大, 而且其线性增大的斜率与潜深之间近似满足指数衰减关系. 特别地, 本 文工作表明，现有文献认为尾迹效应内波关于轴对称回转体纵剖面是对称的先验假定是不合理的.
\end{abstract}

关键词密度分层流体, 轴对称回转体, 体积效应内波, 尾迹效应内波, 反对称度

在不同海洋深度处, 由于温度和盐度的差异导 致了密度的差异, 进而在一定深度上存在着密度梯 度变化的密跃层 ${ }^{[1]}$. 当水下物体在密度垂向层化的海 洋中航行时, 艇体及其尾迹的扰动会使海水微团偏 离其平衡位置进而形成内波 ${ }^{[2]}$. 内波诱导的流场会改 变海洋背景流动的性质, 这不仅会影响海洋中的混 合和能量输运特性 ${ }^{[3]}$, 而且也使得对水下物体航行踪 迹进行非声探测成为一种潜在的军事手段 ${ }^{[4]}$. 因此, 对密度分层流体中水下运动物体产生内波特性问题 的研究, 不仅对物理海洋学有重要的学术意义, 而且 对非声反潜具有重要的潜在军事应用价值.

当水下物体在密度分层流体中运动时，流体翻 越物体表面形成回流区. 无论是物体本身还是附着
于其表面的回流区，都会产生排水体积效应，使海水 微团偏离其平衡位置进而激发内波，这类内波称为 体积效应内波. 由于这两类体积效应源的运动相对 于物体的运动都是定常的, 因此体积效应内波相对 于物体的运动也是定常的. 由此可见，体积效应内波 的生成机理与经典Kelvin波类似，但体积效应内波存 在多种模态的波系结构, 而且其张角随物体运动速 度及密度分层参数而变化, 而经典Kelvin波只有一种 模态的波系结构, 而且张角与航速无关 ${ }^{[2]}$.

密度分层流体中水下运动物体尾迹的形成过程 包括 3 个阶段, 分别为近尾迹阶段、塌陷阶段和晚尾 迹阶段. 近尾迹特性与密度均匀流体的情况类似, 包 括对称浴结构、非对称浴结构和完全湍流等 ${ }^{[5,6]}$. 尾迹

引用格式: 王宏伟, 陈科, 尤云祥, 等. 密度分层流体中不同潜深拖曳回转体激发内波特性实验. 科学通报, 2017, 62: 2132-2148 Wang H W, Chen K, You Y X, et al. Experiments on internal waves generated by a towed revolution body with different depths in a stratified fluid (in Chinese). Chin Sci Bull, 2017, 62: 2132-2148, doi:10.1360/N972016-00864 
塌陷与密度分层流体的浮力效应有关, 其演化特征 是垂向增长受到抑制, 而主要表现为水平方向的扩 散 ${ }^{[7]}$. 晚尾迹的主要特征为水平空间大而垂向厚度相 对较簿的饼状涡街结构, 也称为大尺度湍流相干结 构 $^{[8,9]}$. 近尾迹中的各种不稳定结构、尾迹塌陷激励以 及晚尾迹中的饼浴激励等也会激发内波 ${ }^{[10 ~ 12]}$. 由于 尾迹的生成和演化是一个复杂的随机过程, 因此这 是一类非确定性的内波, 将其称为尾迹效应内波.

如何定量表征体积效应和尾迹效应内波之间的 转换关系, 无论在学术上还是在实践中都是一个重 要的问题. 为此, 定义 Froude数为 $F r=U /\left(D N_{\text {max }}\right)$, 式 中 $U$ 和 $D$ 分别是物体运动速度和最大回转直径, $N_{\max }$ 为密度分层流体的最大浮频率. 在早期, 针对线性密

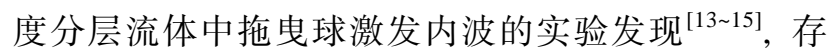
在一个临界转捩Froude数 $F r_{\mathrm{c}} \approx 2.0$, 当 $F r<F r_{\mathrm{c}}$ 时, 体 积效应内波为主控内波; 而当 $F r>F r_{\mathrm{c}}$ 时, 尾迹效应内 波为主控内波. 其后, Robey ${ }^{[16]}$ 和魏岗等人 ${ }^{[17]}$ 针对拖 曳球激发内波的实验表明, 当 $F r<F r_{\mathrm{c}}$ 时, 体积效应内 波的峰-峰幅值与 $F r$ 之间为非线性关系, 而且在某个 Froude数 $F r_{\mathrm{p}}$ 处达到最大值; 当 $F r>F r_{\mathrm{c}}$ 时, 尾迹效应 内波的相关速度会发生突然下降的现象，其相应的 Froude数 $F r_{\mathrm{iw}} \approx 0.8$, 而且内波峰-峰幅值随 $F r$ 近似线 性增加. 此外, 赵先奇等人 ${ }^{[18]}$ 与王进等人 ${ }^{[19,20]}$ 的实 验研究结果表明, 对于细长回转体, 其转㧖点 $F r_{\mathrm{c}}$ 还 与长径比 $\lambda$ 有关.

在水下运动物体激发内波的理论研究方面，早 期主要针对体积效应内波的运动学特性, 将其简化 为移动奇性源产生的波动问题进行研究, 包括射线 理论、驻相法和远场渐近法等 ${ }^{[21 ~ 24]}$. 由于移动奇性源 不能定量表征物体及其回流区产生的排水效应, 难 以获得内波位移场的时空分布等定量信息. 为克服 这种缺陷, 将水下运动物体及其回流区等效为一个 移动柱型质量源, Robey ${ }^{[16]}$ 建立了体积效应内波的一 个理论预测模型, 尤云祥等人 ${ }^{[25]}$ 采用回转椭球体型 等效质量源, 对该方法进行了改进. 针对尾迹效应内 波, 大尺度相干结构激发的内波是一类主控内波 ${ }^{[26]}$. 基于这个事实, 将大尺度相干结构等效为一个移动 柱型质量源, 并结合实验获得的尾迹效应内波相关 速度等信息, Robey ${ }^{[16]}$ 和尤云祥等人 ${ }^{[25]}$ 的研究进一步 表明, 所建立的等效质量源理论模型可以用于尾迹 效应内波特性的理论预测.

需要指出的是, 目前有关水下运动物体激发内
波特性研究尚有不足之处. (1) 目前的实验结果均是 在物体位于某一个固定潜深的情况下获得的, 然而, 有关潜深对两类内波之间的转捩特性及其位移场时 空分布特征的影响等问题, 迄今尚不清楚. (2) 目前 关于水下运动物体激发内波的相关理论模型及实验 研究中, 都先验地假定一个轴对称回转体激发的内 波关于物体中纵剖面是对称的. 在理论模型研究中, 移动奇性源(点源、偶极子, 以及等效质量源等)的设 置都是轴对称的, 而在实验研究中, 通常只测量水槽 中纵剖面一侧的内波信息，而另一侧的内波信息是 通过对称方法获得的，但是，尾迹效应内波含有明显 随机湍流成分, 使用对称假设进行研究, 可能是不合 理的.

有鉴于此, 本文以某长径比为 7.7 的轴对称回转 体模型为对象, 利用重力式密度分层水槽, 采用沿水 槽横向整体布置电导率探头阵列的技术, 结合相关 性分析和奇偶函数分解等方法，在 5 个不同潜深下, 对该水下拖曳模型在具有密度跃层分层流体中激发 体积效应和尾迹效应内波的时空特性开展系列实验, 重点研究两类内波的转捩特性、位移场特性、时空形 态特征及对称性等问题.

\section{1 实验系统与方法}

实验是在解放军理工大学重力式分层流水槽系 统中进行，该系统由水槽主体、分层流制取系统、循 环拖曳系统、内波动态测量系统和实验模型等组成, 如图1所示. 水槽主尺度为 $12 \mathrm{~m} \times 1.2 \mathrm{~m} \times 1 \mathrm{~m}$ (长×宽 $\times$ 高), 分层流制取系统由两个体积均为 $2.2 \mathrm{~m} \times 2.2$ $\mathrm{m} \times 1.6 \mathrm{~m}$ (长 $\times$ 宽 $\times$ 高 $)$ 的水箱通过流量可控的管道与水 槽底部两个蘑菇型进水口相连, 箱底距地面 $2.36 \mathrm{~m}$,

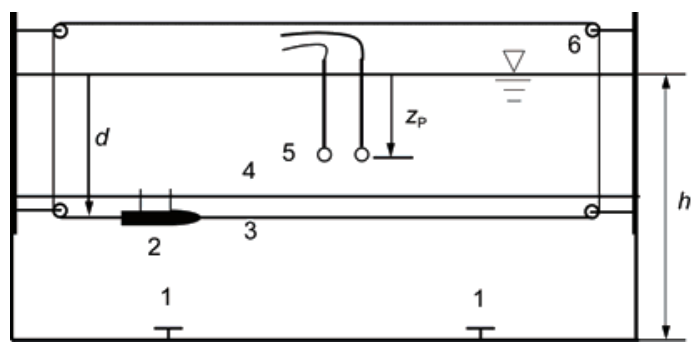

图 1 实验系统示意图(侧视). 1, 蘑菇型进水口; 2, 拖曳细长体; 3, 拖曳线; 4 , 引导线; 5 , 电导率探头阵列; 6 , 伺服电机

Figure 1 Schematic of experimental system (side view). 1, Mushroomlike inlet; 2 , towed slender body; 3 , towing wire; 4 , guiding wire; 5 , conductivity probe arrays; 6 , servo system 
可通过重力式方法形成线性密度分层、强跃层分层等 密度分层流体. 水槽底部两个蘑菇型进水口分别安 装在距水槽两端 $1 / 4$ 和 $3 / 4$ 长度处, 这种方式可使盐水 缓慢、均匀地注人水槽.

实验模型为直径 $D=5 \mathrm{~cm}$ 和长径比 $\lambda=L / D=7.7$ 的 细长回转体, 采用双线循环拖曳法驱动实验模型, 将 其中心系于直径为 $0.18 \mathrm{~cm}$ 的细钢丝绳上作为循环拖 曳线, 并在其上方用同样直径的张紧式钢丝绳作为 引导线, 以防止模型在拖电过程中的晃动, 拖曳线通 过固定滑轮连接到外部伺服电机上, 可以实现调速 范围0 2 $\mathrm{m} / \mathrm{s}$ 的拖曳速度.

内波动态测量系统由多通道电导率探头阵列、电 导率采集放大系统、 $\mathrm{A} / \mathrm{D}$ 转换系统和数据实时采集处 理系统等组成. 通过实验前电导率与密度的标定公 式可将电导率换成密度值, 该系统既可用于密度随 时间变化的动态测量, 也可用于密度剖面的静态测 量. 探头采样频率为 $20 \mathrm{~Hz}$, 响应时间为 $5 \mathrm{~ms}$, 远小 于拖曳体激发内波的特征周期.

为下文陈述方便, 建立如图2所示的直角坐标系 $o-x y z$. 其中, $o x y$ 面为静水面, $o x z$ 面为水槽中纵剖面, $o y z$ 面为水槽中横剖面, $o z$ 轴垂直向下为正. 在实验 中, 布置两组电导率探头阵列, 第一组在 $(x, z)=\left(0, z_{\mathrm{P}}\right)$ 处沿着水槽 $y$ 方向对称布置 23 个电导率探头, 用 $P_{0}$, $P_{ \pm 1}, \cdots, P_{ \pm 11}$ 对各探头进行编号, 每个探头沿 $y$ 方向间 隔 $\Delta y_{1}=5 \mathrm{~cm}$, 探头 $P_{0}$ 在水槽横向正中间处. 该组电导 率探头阵列用来测量模型运动产生内波的时空特征. 第二组在 $(x, z)=\left(\Delta x, z_{\mathrm{P}}\right)$ 处沿着水槽 $y$ 方向布置 4 个电导 率探头, 分别与第一组的 $P_{+1}, P_{+3}, P_{+5}$ 和 $P_{+7}$ 探头对应, 将其分别编号为 $Q_{+1}, Q_{+3}, Q_{+5}$ 和 $Q_{+7}$, 各探头沿 $y$ 方向 间隔 $\Delta y_{2}=10 \mathrm{~cm}$, 并与第一组探头在 $x$ 方向的间

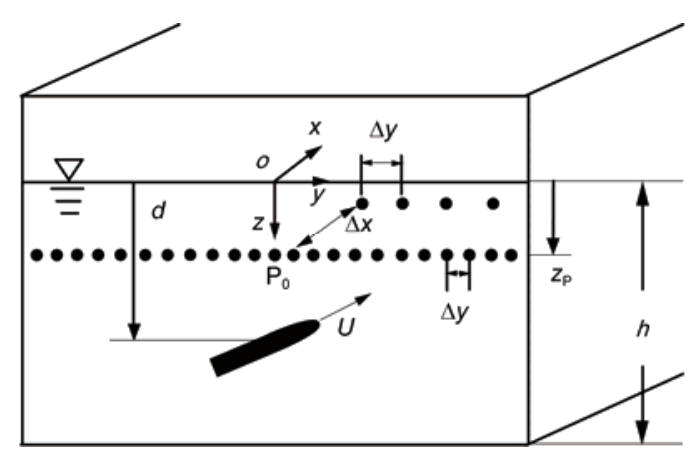

图 2 坐标系及探头布置示意图

Figure 2 Schematic of the coordinate system and arrangement of probes
距为 $\Delta x=176 \mathrm{~cm}$. 该组探头用来测量分析模型运动产 生内波沿 $o x$ 轴方向传播的相关速度.

本文实验采用强跃层分层流体. 实验中, 首先注 人厚度 $h_{1}=30 \mathrm{~cm}$ 和密度 $\rho_{1}=998 \mathrm{~kg} / \mathrm{m}^{3}$ 的淡水, 静置一 段时间后, 通过水槽底部的两个蘑菇型进水口缓慢地 注人密度 $\rho_{2}=1023 \mathrm{~kg} / \mathrm{m}^{3}$ 的盐水, 直到总水深 $h=80 \mathrm{~cm}$. 利用电导率探头, 采用垂直下降的方式, 每间隔 0.5 $\mathrm{cm}$ 测量一次电导率数据, 再在深度上每间隔 $0.1 \mathrm{~cm}$ 进行插值, 得到垂向密度剖面及浮力频率剖面, 结果 如图3所示. 其中, 浮力频率 $N(\mathrm{z})=\sqrt{-(g / \rho)(\partial \rho / \partial \mathrm{z})}$, 式中 $\rho$ 为密度垂向分布, $g$ 为重力加速度. 另外, 本文 $z$ 轴设定为方向向下, 故实际计算时, 不计算根号内的 负号. 图3中, 实线为首次实验时的剖面, 虚线为最 后一次实验时的剖面. 可知, 最大浮力频率在深度 $z=29 \mathrm{~cm}$ 附近, 且 $N_{\max }$ 在 $1.54 \sim 1.92 \mathrm{rad} / \mathrm{s}$ 范围内变化. 不过, 在无因次化分析中考虑 $N_{\text {max }}$ 可消除其变化带来 的影响.

海上现场观测和数值模拟结果表明, 内波在最 大浮力频率所在水平层的稍下方处振幅最大 ${ }^{[27]}$, 因 此将电导率探头置于最大振幅位置为最佳选择. 不 过, 由于此位置的精确值较难获得, 实验研究中, 一

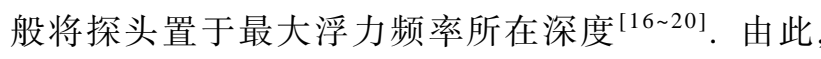
本文实验中, 将各电导率探头置于 $z_{\mathrm{P}}=29 \mathrm{~cm}$ 处, 重点 研究该水平层模型运动激发内波特性. 将实验模型 置于最大浮力频率所在水平层下方, 选取 5 个不同潜 深, 分别为 $d=35,38,41,47$ 和 $53 \mathrm{~cm}$. 在每个潜深下, 拖曳速度 $U$ 的范围为 $5 \sim 160 \mathrm{~cm} / \mathrm{s}$, 对应的Froude数 $F r=U / D N_{\text {max }}$ 的范围为 $0.3 \leqslant F r \leqslant 16.7$, Reynold数 $R e=$ $U D / v$ 的范围为 $2500 \leqslant R e \leqslant 80000$. 其中, $v=10^{-2} \mathrm{~cm}^{2} / \mathrm{s}$ 为盐水的动力学黏性系数. 在每次实验后, 均等待 20 $\min$ 左右, 然后再做下一个工况的实验.

\section{2 结果与分析}

\section{1 内波转捩特性}

体积效应和尾迹效应内波之间的转捩特性，可以 通过测量其相关速度 $U_{\mathrm{iw}}$ 进行研究. 关于内波速度的 说法较多, 包括内波波致流速、线性相速度和非线性 相速度等 ${ }^{[28]}$, 本节参照Robey ${ }^{[16]}$ 对于相关波速的测 量方法, 设 $P$ 和 $Q$ 是位于最大浮力频率所在水平层的 两个电导率探头, 其沿水槽横向坐标 $y$ 相同, 纵向间 距为 $\Delta x$. 对其测量得到的密度扰动信号进行相关性分 


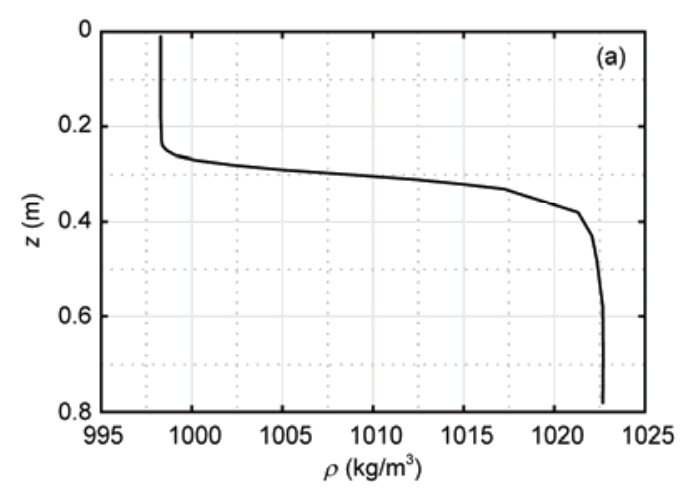

图 3 密度(a)及浮力频率(b)剖面

Figure 3 Profiles of density (a) and buoyancy frequency (b)

析, 相关性峰值对应的时间记为 $\Delta t$, 此即内波从起始 探头 $P$ 传到另一个探头 $Q$ 所需的时间. 由此可得, 拖 曳模型产生内波的相关速度为 $U_{\mathrm{iw}}=\Delta x / \Delta t$. 对直径 $D$ 的拖曳潜体, 当最大浮力频率为 $N_{\max }$ 时, 可进一步将 内波相关速度无量纲化为相关速度Froude数 $F r_{\mathrm{iw}}$ 如 下:

$$
F r_{\text {iw }}=U_{\text {iw }} / D N_{\text {max }} .
$$

在已有文献的报道中 ${ }^{[13 \sim 15]}$, 通常认为在拖电模 型激发的内波发生转捩前, 体积效应内波为主控内 波, 内波相关速度 $U_{\mathrm{iw}}$ 与拖曳速度 $U$ 一致; 转捩后, 尾 迹效应内波为主控内波, $U_{\mathrm{iw}}$ 出现突然下降的现象, 并在某个常数附近小幅波动. Robey ${ }^{[16]}$ 认为可用临界 Froude数 $F r_{\mathrm{c}}$ 来界定内波是否转捩, 即当Froude数 $F r>F r_{\mathrm{c}}$ 时, 内波发生转捩, 而且对于拖曳球体, 有 $F r_{\mathrm{c}}=2$. 王进等人 ${ }^{[19,20]}$ 进一步研究了 4 个不同长径比 $(\lambda=1,4,7$ 和9)的回转体拖曳体激发内波特性, 发现 $F r_{\mathrm{c}}$ 与长径比 $\lambda$ 之间具有如下的关系:

$$
F r_{\mathrm{c}}=0.2391 \lambda+1.7579 \text {. }
$$

本文实验模型的长径比为 $\lambda=7.7$, 本节目的之一 是检验王进等人 ${ }^{[19,20]}$ 所得关系(2)式是否也适用于该 实验模型. 另一方面, 在现有文献的实验中, 仅考虑 了水槽某个纵剖面上的内波相关速度特性, 那么在 水槽不同纵剖面上的内波相关速度是否也具有这样 的特性, 目前尚不清楚. 为此, 本节目的之二是通过 在水槽横向 4 个不同位置前后各布置两个电导率探头 的方式, 研究在水槽不同纵剖面上的内波相关速度 特性. 另外, 目前关于两类内波转捩特性实验, 仅考 虑了某一个潜深的情况, 但关于内波转捩特性与潜 深之间的相关关系, 目前也尚不清楚. 为此, 本节目 的之三是针对 5 个不同潜深, 研究拖曳模型产生内波

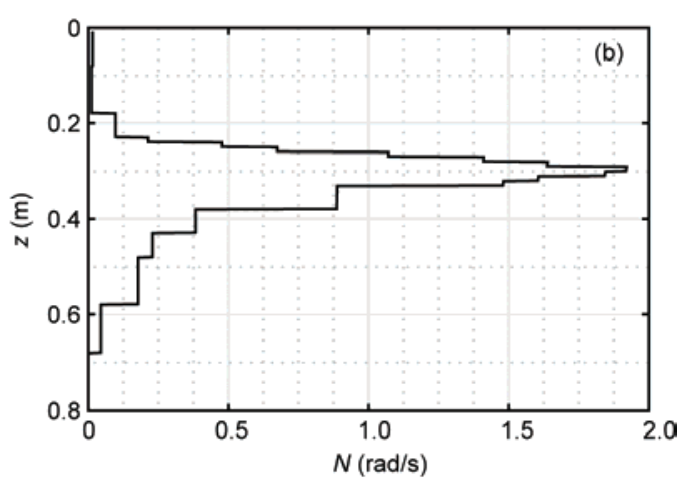

的转㧖特性与潜深之间的相关关系.

利用4组电导率探头 $P_{+1}-Q_{+1}, P_{+3}-Q_{+3}, P_{+5}-Q_{+5}$ 和 $P_{+7} Q_{+7}$, 测量拖曳潜体产生的密度扰动信号, 分别 对其进行相关性分析，进而获得在水槽横向 4 个不同 纵向剖面上内波的相关速度, 研究拖曳潜体产生内 波在不同潜深下的转捩特性, 结果如图4所示.

由图可知, 对 5 个不同的潜深, 均存在一个近似 相同的临界Froude数 $F r_{\mathrm{c}}=3.64$, 当 $F r<F r_{\mathrm{c}}$ 时, 在不同 纵剖面上, 内波相关速度 $U_{\mathrm{iw}}$ 均与模型拖曳速度 $U$ 一 致, 表明拖曳模型激发内波相对拖曳体的运动是定 常的, 此时体积效应内波为主控内波; 当 $F r>F r_{\mathrm{c}}$ 时, 在不同纵剖面上, 内波相关速度均出现突然下降的 现象, 表明拖曳模型激发内波相对拖曳体的运动是 非定常的, 此时尾迹效应内波为主控内波. 结果进一 步表明, 对 5 个不同潜深, 转捩后在不同纵剖面上内 波相关速度下降的幅度有较大差异, 而且随着Froude 数 $F r$ 的变化, 内波相关速度Froude数 $F r_{\text {iw }}$ 并没有出现 在某个常数附近小幅波动的现象, 而是在0.7 1.4之 间的一个条带内变化, 条带均值约为 1.0 , 变化幅度 达0.7.

将本文实验模型的长径比 $\lambda=7.7$ 代人(2)式，可得 内波临界Froude数的经验预测值为 $F r_{\mathrm{c}}=3.60$, 这与本 文实验所得 $F r_{\mathrm{c}}=3.64$ 一致. 由此可见, 对内波临界 Froude数 $F r_{\mathrm{c}}$ 经验预测公式(2), 不仅适用于不同长径 比回转体的情况, 而且适用于不同潜深的情况. 这为 预测水下航行体在实际密度分层海洋中航行时激发 内波的转捩特性提供了一个实用的方法.

在图5中, 给出了南海某海域春夏秋冬四季浮力 频率的实测结果. 由图可知, 春季最大浮力频率位于 水面下 $39.2 \mathrm{~m}$ 处, $N_{\max }=1.90 \times 10^{-2} \mathrm{rad} / \mathrm{s}$; 夏季最大浮 

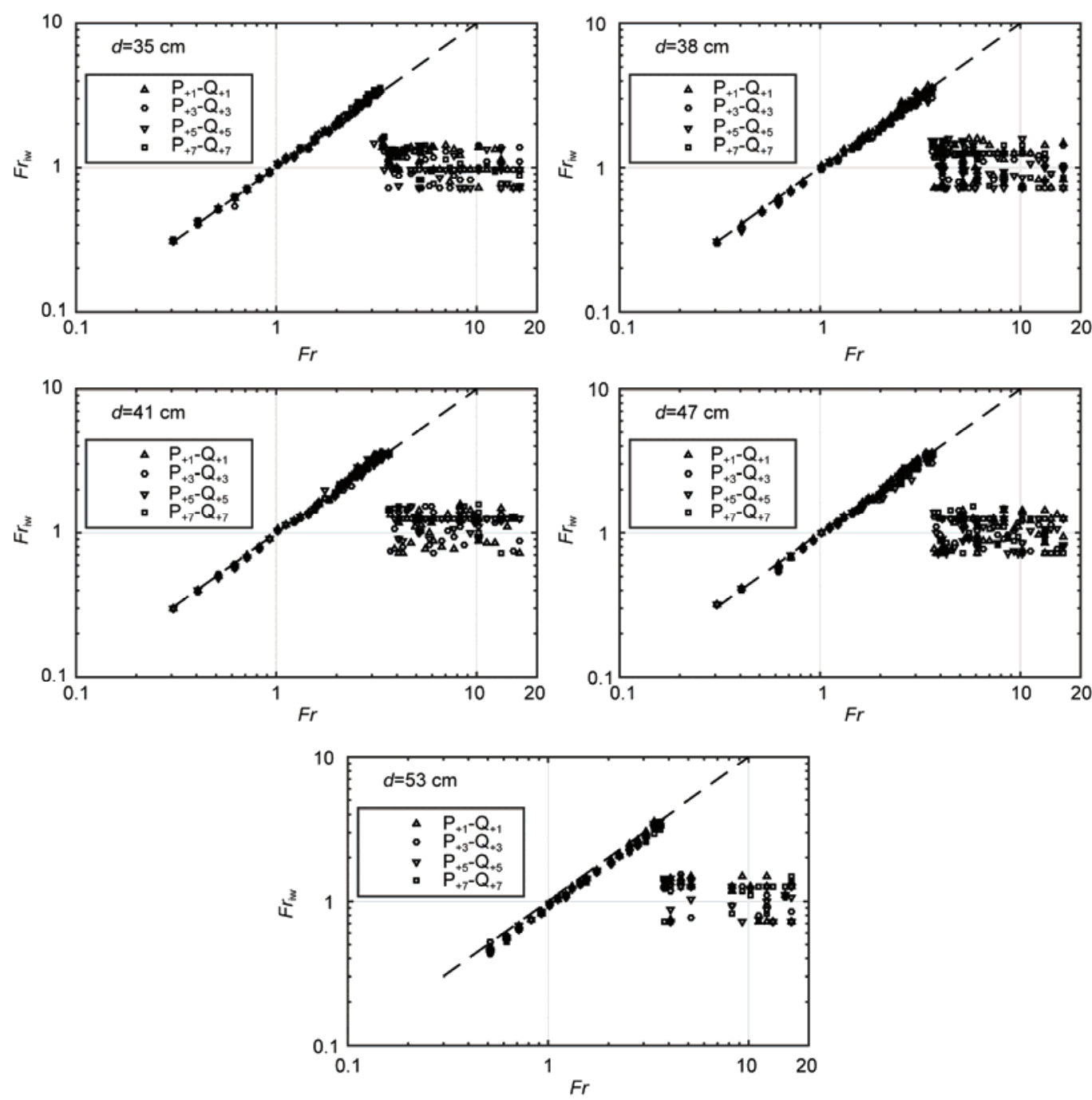

图 $4 F r_{\text {iw }}$ 随 $F r$ 的变化规律

Figure 4 Correlation velocity Froude numbers $F r_{\text {iw }}$ versus towing Froude numbers $F r$

力频率位于水面下 $44.1 \mathrm{~m}$ 处, $N_{\text {max }}=2.87 \times 10^{-2} \mathrm{rad} / \mathrm{s}$, 与春季的情况相比, 夏季主跃层深度要大一些, 而且 最大浮力频率也要大一些; 秋季最大浮力频率位于 水面下 $62.4 \mathrm{~m}$ 处, $N_{\text {max }}=2.29 \times 10^{-2} \mathrm{rad} / \mathrm{s}$, 与春夏季的 情况相比, 秋季主跃层深度要更深一些; 冬季最大浮 力频率位于水面下 $94.3 \mathrm{~m}$ 处, $N_{\max }=1.62 \times 10^{-2} \mathrm{rad} / \mathrm{s}$, 与其他季节的情况相比, 冬季主跃层深度要大很多, 可达90余米.

假设水下航行体长径比为 $\lambda=10$, 回转直径 $D$ 为 $12 \mathrm{~m}$. 由(2)式可得, 该水下航行体的内波临界转捩 Froude数 $F r_{\mathrm{c}}=4.15$. 由此可得, 水下航行体在春季的 临界航速为 $U_{\mathrm{c}}=F r_{\mathrm{c}} D N_{\max }=0.95 \mathrm{~m} / \mathrm{s}$; 在夏季的临界航 速为 $U_{\mathrm{c}}=1.43 \mathrm{~m} / \mathrm{s}$; 在秋季的临界航速为 $U_{\mathrm{c}}=1.14 \mathrm{~m} / \mathrm{s}$;
在冬季的临界航速为 $U_{\mathrm{c}}=0.90 \mathrm{~m} / \mathrm{s}$. 由于水下航行体 在巡航状态下的航速一般为 $4 \mathrm{kn}(\approx 2.0 \mathrm{~m} / \mathrm{s})$, 大于各 季节下的临界航速 $U_{\mathrm{c}}$, 因此在实际海洋中, 水下航行 体激发的内波主要以尾迹内波为主控内波.

对尾迹内波，由前面的实验结果可知，其相关速 度Froude数 $F r_{\mathrm{iw}}$ 介于 $0.7 \sim 1.4$ 之间. 由(1)式可得, 内波 相关速度为 $U_{\mathrm{iw}}=F r_{\mathrm{iw}} D N_{\max }$. 由此可得，水下航行体 尾迹内波在春季的相关速度介于 $0.16 \sim 0.32 \mathrm{~m} / \mathrm{s}$; 在夏 季的相关速度介于 $0.24 \sim 0.48 \mathrm{~m} / \mathrm{s}$; 在秋季的相关速度 介于 $0.19 \sim 0.38 \mathrm{~m} / \mathrm{s}$; 在冬季的相关速度介于 $0.14 \sim$ $0.28 \mathrm{~m} / \mathrm{s}$.

由此可见，在实际海洋中水下航行体激发内波 的相关速度要远小于其航速. 这意味着水下航行体 

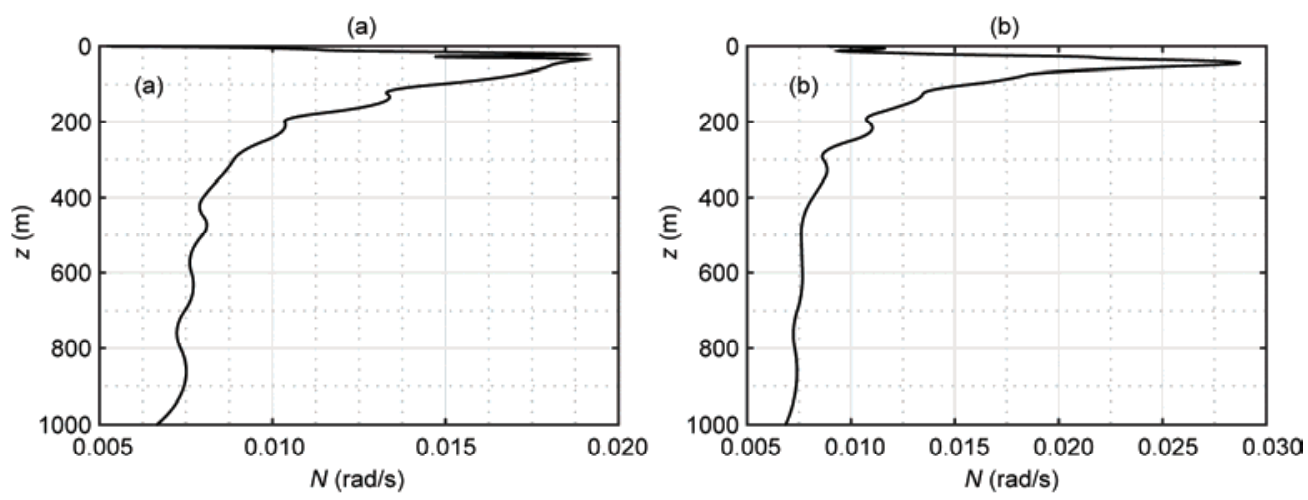

(c)
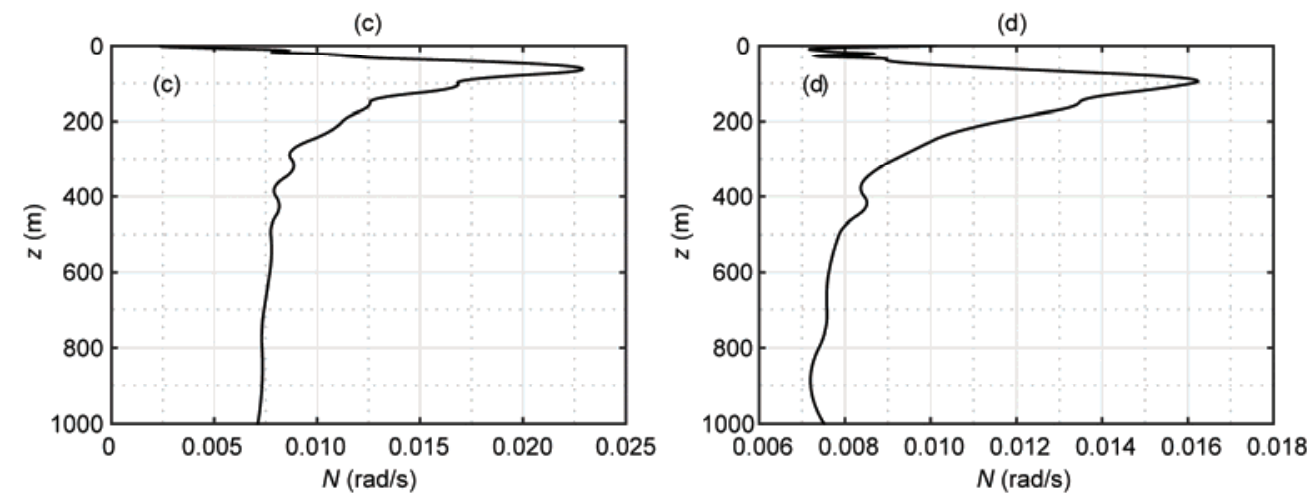

图 5 南海某海域春夏秋冬四季浮力频率测量结果. (a) 春季; (b) 夏季; (c) 秋季; (d) 冬季

Figure 5 Profiles of buoyancy frequency measured in the South China Sea in four seasons. (a) Spring; (b) summer; (c) autumn; (d) winter

经过后, 沿其航迹在其尾部会留下很长的内波尾迹 信息. 由于内波的恢复力很小，因此内波尾迹在水下 航行体航迹上会滞留很长的时间. 这为通过内波尾 迹信息对水下航行体的航行踪迹进行跟踪和监测提 供了一定的理论依据.

\section{2 内波位移场特性}

记 $\Delta \rho=\Delta \rho\left(x_{0}, y_{0}, z_{0}, t\right)$ 为在点 $\left(x_{0}, y_{0}, z_{0}\right)$ 处密度扰动与 该点处基准密度之差的时历, 那么在该点处密度波

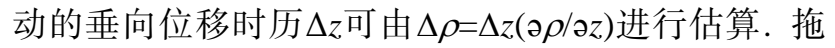
曳模型产生内波的位移场特性是理论和实践中都关

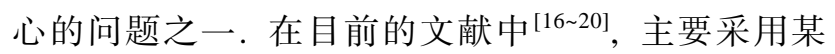
个传感器处内波位移时历的峰-峰幅值, 对拖曳模型 产生内波的位移场特性进行分析, 但这种处理方法 有其局限性.

在图6中, 给出了潜深 $d=35 \mathrm{~cm}$ 时在 $P_{ \pm 3}$ 处拖曳模 型产生内波位移的时历特性. 由图可知, 在转捩前后 及不同位置处, 内波峰-峰值出现的时刻并不一致, 而且转㧖后内波位移场关于水槽中纵剖面并不对称. 因此, 在目前文献中关于拖曳模型产生内波位移场
波高特性的统计方法，并不能反映其整体的时间和 空间变化特征及规律.

本文电导率仪的采样频率为 $20 \mathrm{~Hz}$ ，即采样时间 间隔为 $\Delta t=0.05 \mathrm{~s}$. 对每个电导率探头采集到的密度 扰动信号取其前 $100 \mathrm{~s}$ 的结果, 总的采样点为 $N=2001$. 将采集到的密度扰动信号换算为相应的内波位移信 号, 设 $X_{1}, X_{2}, \cdots, X_{N}$ 为监测点 $\left(0, y, z_{\mathrm{P}}\right)$ 处拖曳模型产生 内波位移的时间序列, 取其根均方差 (Root Mean Square Deviation, RMSE) $S$ 如下:

$$
S=S(y)=\sqrt{\frac{1}{N-1} \sum_{i=1}^{N}\left(X_{i}-\bar{X}\right)^{2}},
$$

其中, $\bar{X}$ 为时间序列的均值.

在图7中, 给出了转捩前无量纲根均方差 $S / S_{0}$ 随 $y$ 变化特性的结果, 其中 $S_{0}=S(0)$. 由图可知, 在 -0.25 $\mathrm{m}<y<0.25 \mathrm{~m}$ 范围内, 在不同拖曳速度组合下, $S / S_{0}$ 随 $y$ 变化的散点图近似与高斯分布一致, 图中实线为 $B=0.15 \mathrm{~m}$ 的如下高斯函数:

$$
S(y) / S_{0}=\mathrm{e}^{-y^{2} / 2 B^{2}} .
$$

但在 $-0.25 \mathrm{~m}<y<0.25 \mathrm{~m}$ 范围外, $S / S_{0}$ 随 $y$ 变化的散 点图与高斯分布偏离很大, 这主要与水槽侧壁导致 

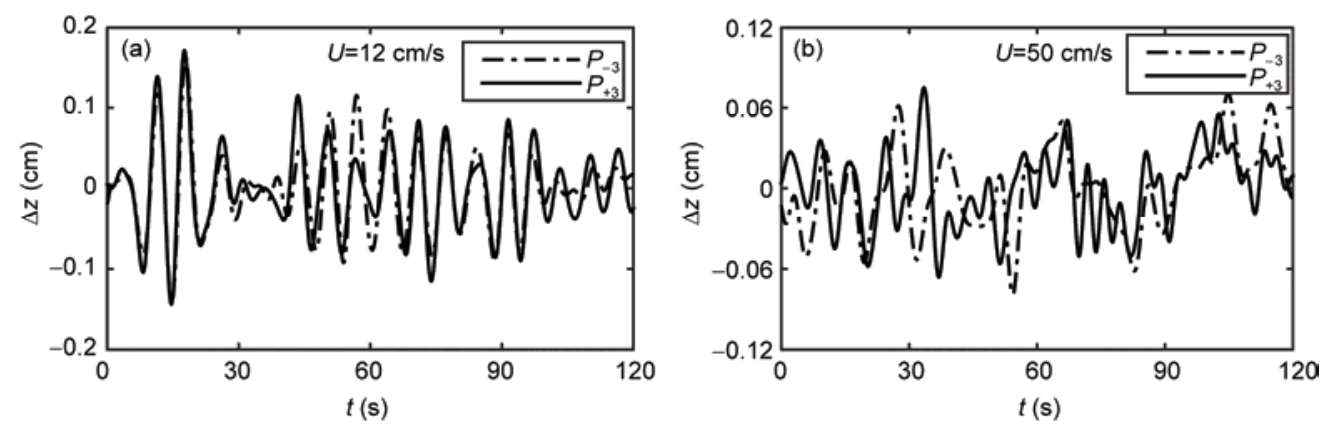

图 6 潜深 $d=35 \mathrm{~cm}$ 时, 内波位移时历特性. (a) $U=12 \mathrm{~cm} / \mathrm{s}$ (转捩前); (b) $U=50 \mathrm{~cm} / \mathrm{s}$ (转㧖后)

Figure 6 Time series of internal wave vertical displacement when $d=35 \mathrm{~cm}$. (a) $U=12 \mathrm{~cm} / \mathrm{s}$ (before transition); (b) $U=50 \mathrm{~cm} / \mathrm{s}$ (after transition)

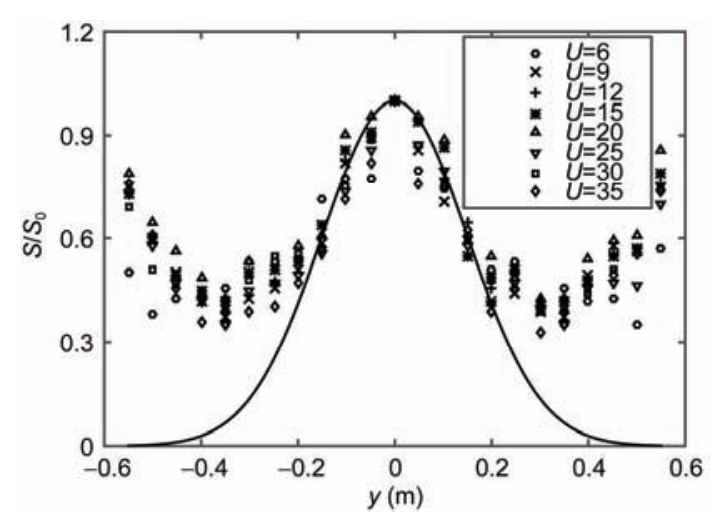

图 7 潜深 $d=35 \mathrm{~cm}$ 时, 转捩前 $S / S_{0}$ 随 $y$ 轴分布

Figure 7 Distribution of $S / S_{0}$ along $y$-axis before transition when $d=$ $35 \mathrm{~cm}$

的内波反射有关. 由此可见, 在前 $100 \mathrm{~s}$ 的时间内, 拖 曳模型产生内波在 $-0.25 \mathrm{~m}<y<0.25 \mathrm{~m}$ 范围内, 受水槽 侧壁的影响较小. 由图还可进一步发现, 在转捩前, $S / S_{0}$ 在水槽中纵剖面 $(y=0)$ 处达到最大值; 在较小拖 曳速度下, $S / S_{0}$ 关于 $y$ 是对称的, 而且其散点图与高斯 分布的偏差很小, 这表明此时尾迹效应内波的影响 很小, 拖曳模型产生内波主要表现为体积效应内波; 随着拖电速度的增大, $S / S_{0}$ 关于 $y$ 的对称性随着变差, 而且其散点图与高斯分布的偏差也随着变大, 这表 明虽然此时体积效应内波仍为主控内波, 但尾迹效 应内波的影响逐渐增大.

在图8中, 给出了转捩后无量纲根均方差 $S / S_{0}$ 随 $y$ 变化特性的实验结果. 由图可知, 在转捩后, $S / S_{0}$ 关 于 $y$ 并不是对称的, 而且 $S / S_{0}$ 并不一定在水槽中纵剖 面 $(y=0)$ 处达到最大值; 与转㧖前的情况类似, 在 $-0.25 \mathrm{~m}<y<0.25 \mathrm{~m}$ 范围外, 拖曳模型产生内波受水槽 侧壁反射的影响显著; 随着拖曳速度的增大, 在 $-0.25 \mathrm{~m}<y<0.25 \mathrm{~m}$ 范围内, $S / S_{0}$ 的峰一峰幅值也随着增
大, 但峰和谷出现的位置具有随机性. 由此可见, 在 转捩后, 主控内波尾迹效应内波具有明显的随机性, 而且关于水槽中纵剖面 $(y=0)$ 并不是对称的.

在实践中, 内波峰-峰幅值特性是一个重要的参 数. 由于水下航行体激发内波关于其中纵剖面一般 是不对称的, 因此如果仅用某一个电导率探头所得 内波的峰-峰幅值，难以反映其在空间上的全局特性. 为此, 根据上述密度波动的根均方差在横剖面上的 分布特性, 采用如下方法统计内波的峰-峰幅值: 在 $-0.25 \mathrm{~m}<y<0.25 \mathrm{~m}$ 范围内, 对9个电导率探头 $P_{0}, P_{ \pm 1}$, $P_{ \pm 2}, P_{ \pm 3}$ 和 $P_{ \pm 4}$ 所得内波位移时历的峰-峰幅值进行统 计, 记其最大峰- 峰幅值为 $H_{\mathrm{m}}$, 取其无量纲形式为 $H_{\mathrm{m}} / D$.

在图9中, 给出了不同潜深下 $H_{\mathrm{m}} / D$ 随Froude数 $F r$ 的变化规律的实验结果. 由图可知, 在不同潜深下, 转捩前均存在一个近似相同的峰值Froude数 $F r_{\mathrm{p}} \approx$ 1.33, 当 $F r<F r_{\mathrm{p}}$ 时, $H_{\mathrm{m}} / D$ 随 $F r$ 增大而增大, 而当 $F r_{\mathrm{p}}<$ $F r<F r_{\mathrm{c}}$ 时, $H_{\mathrm{m}} / D$ 随 $F r$ 增大而减小; 在转捩后, $H_{\mathrm{m}} / D$ 随 $F r$ 的增大近似线性增大, 但线性增大的斜率 $k_{\mathrm{H}}$ 与潜 深有关.

为定量描述转捩后 $H_{\mathrm{m}} / D$ 随 $F r$ 线性增长的斜率 $k_{\mathrm{H}}$ 随潜深的变化规律, 图 10 给出了 $k_{\mathrm{H}}$ 与 $\Delta d$ 之间的相关 关系的实验结果, 其中 $\Delta d=d-z_{\mathrm{P}}$. 利用图中数据, 采 用数据拟合方法, 可得斜率 $k_{\mathrm{H}}$ 与 $\Delta d / D$ 之间近似满足 如下指数衰减关系:

$$
k_{\mathrm{H}}=0.0176 \mathrm{e}^{-0.418 \Delta d / D} .
$$

由上一节的分析可知, 在实际海洋中水下航行 体激发的内波主要以尾迹内波为主控内波. 因此, 其 内波最大峰-峰幅值与Froude数 Fr之间可以近似用如 下线性关系进行预测

$$
H_{\mathrm{m}} / D=k_{\mathrm{H}} F r
$$



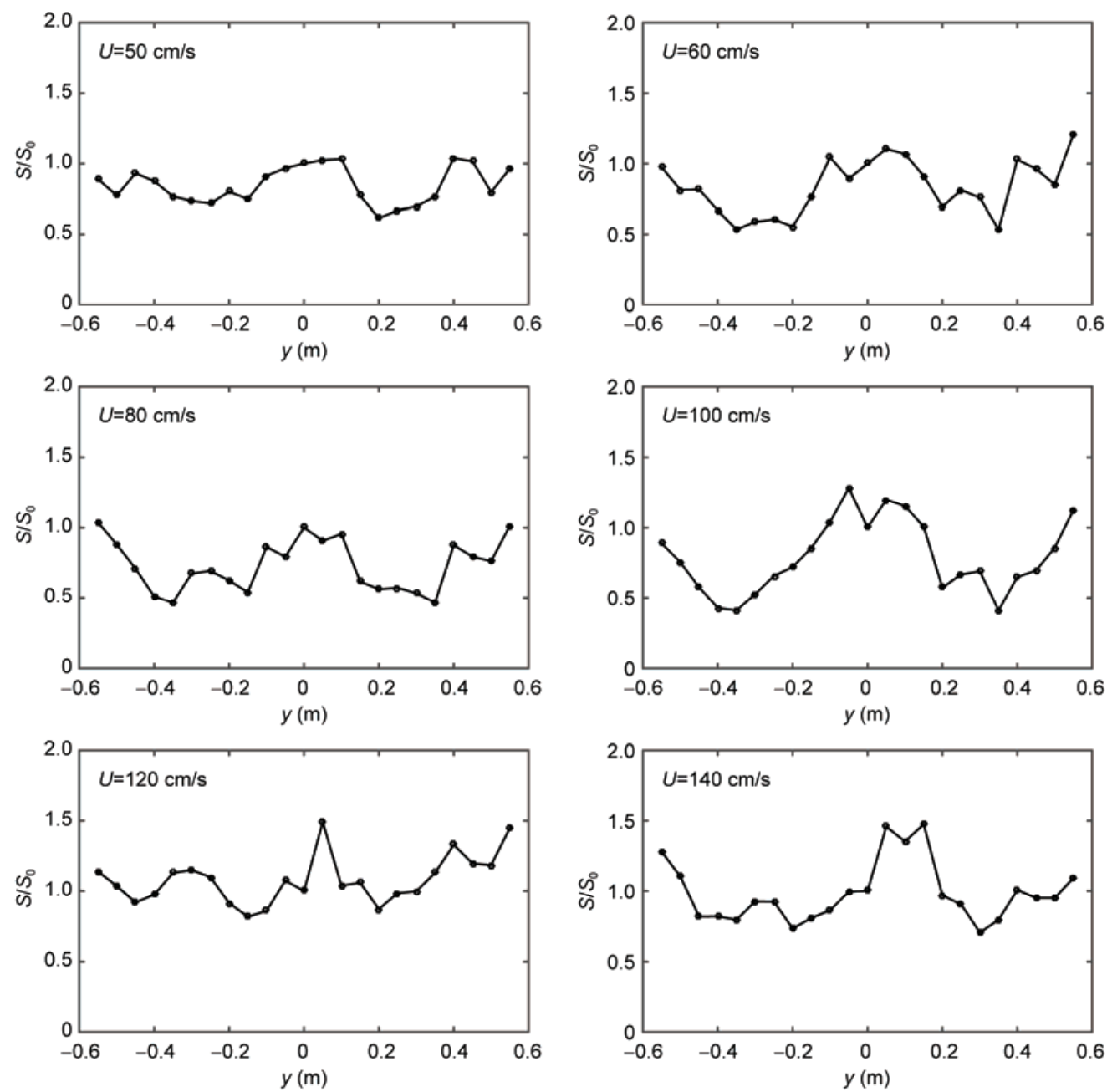

图 8 当潜深 $d=35 \mathrm{~cm}$ 时, 转捩后 $S / S_{0}$ 随 $y$ 轴分布

Figure 8 Distribution of $S / S_{0}$ along $y$-axis after transition when $d=35 \mathrm{~cm}$

王进等人 ${ }^{[19,20]}$ 的研究表明, 对不同长径比拖曳 回转体激发内波, 在转捩后其无量纲最大峰-峰幅值 随 $F r$ 的增大也是近似线性增加的, 而且其线性增加 的斜率与回转体的长径比无关, 斜率 $k_{\mathrm{H}}$ 均近似为一 个常数 0.0124 . 进一步分析可知, 王进等人 ${ }^{[19,20]}$ 采用 的实验模型回转直径为 $D=7 \mathrm{~cm}$, 回转轴到最大浮力 频率所在水平层的距离为 $\Delta d=7 \mathrm{~cm}$, 即 $\Delta d / D=1$. 将其 代人 (5)式可得, $k_{\mathrm{H}}=0.0116$, 这与王进等人 ${ }^{[19,20]}$ 的实 验结果 $k_{\mathrm{H}}=0.0124$ 一致. 由此可见, 本文所得不同潜 深下无量纲最大峰-峰幅值 $H_{\mathrm{m}} / D$ 随Froude数 $F r$ 线性变 化斜率 $k_{\mathrm{H}}$ 的预测模型(5)式，对不同长径比的水下航 行体均是适用的. 这为预测实际海洋中水下航行体 激发内波的最大峰-峰幅值特性, 提供了一定的理论 依据.

水下航行体的航速一般在 4 30 $\mathrm{kn}$ 之间, 即其航 行速度 $U$ 一般在 $2 \sim 15 \mathrm{~m} / \mathrm{s}$ 之间. 由上一小节可知，在 夏季南海密度分层流体的主跃层深度为 $z_{\mathrm{P}}=44.1 \mathrm{~m}$, 最大浮力频率为 $N_{\text {max }}=2.87 \times 10^{-2} \mathrm{rad} / \mathrm{s}$. 由此可见, 对 回转直径为 $D=12 \mathrm{~m}$ 的水下航行体, 其Froude数 $F r$ 的范 围约为 $6 \leqslant F r \leqslant 44$. 由(5)和(6)式可知, 当潜深 $d=50 \mathrm{~m}$ 时, 水下航行体产生内波的最大峰-峰幅值的范围约 为 $1 \mathrm{~m} \leqslant H_{\mathrm{m}} \leqslant 7.6 \mathrm{~m}$; 当潜深 $d=60 \mathrm{~m}$ 时, 水下航行体产 生内波的最大峰-峰幅值的范围约为 $0.7 \mathrm{~m} \leqslant H_{\mathrm{m}} \leqslant 5.3$ $\mathrm{m}$; 当潜深 $d=80 \mathrm{~m}$ 时, 水下航行体产生内波的最大峰峰幅值的范围约为 $0.4 \mathrm{~m} \leqslant H_{\mathrm{m}} \leqslant 2.7 \mathrm{~m}$. 由此可见, 当 水下航行体在密度跃层下航行时, 在巡航状态下, 其 激发内波的幅值很小; 在其高速航行状态下，随着潜 深的增大，其激发内波的幅值呈指数衰减. 这表明, 

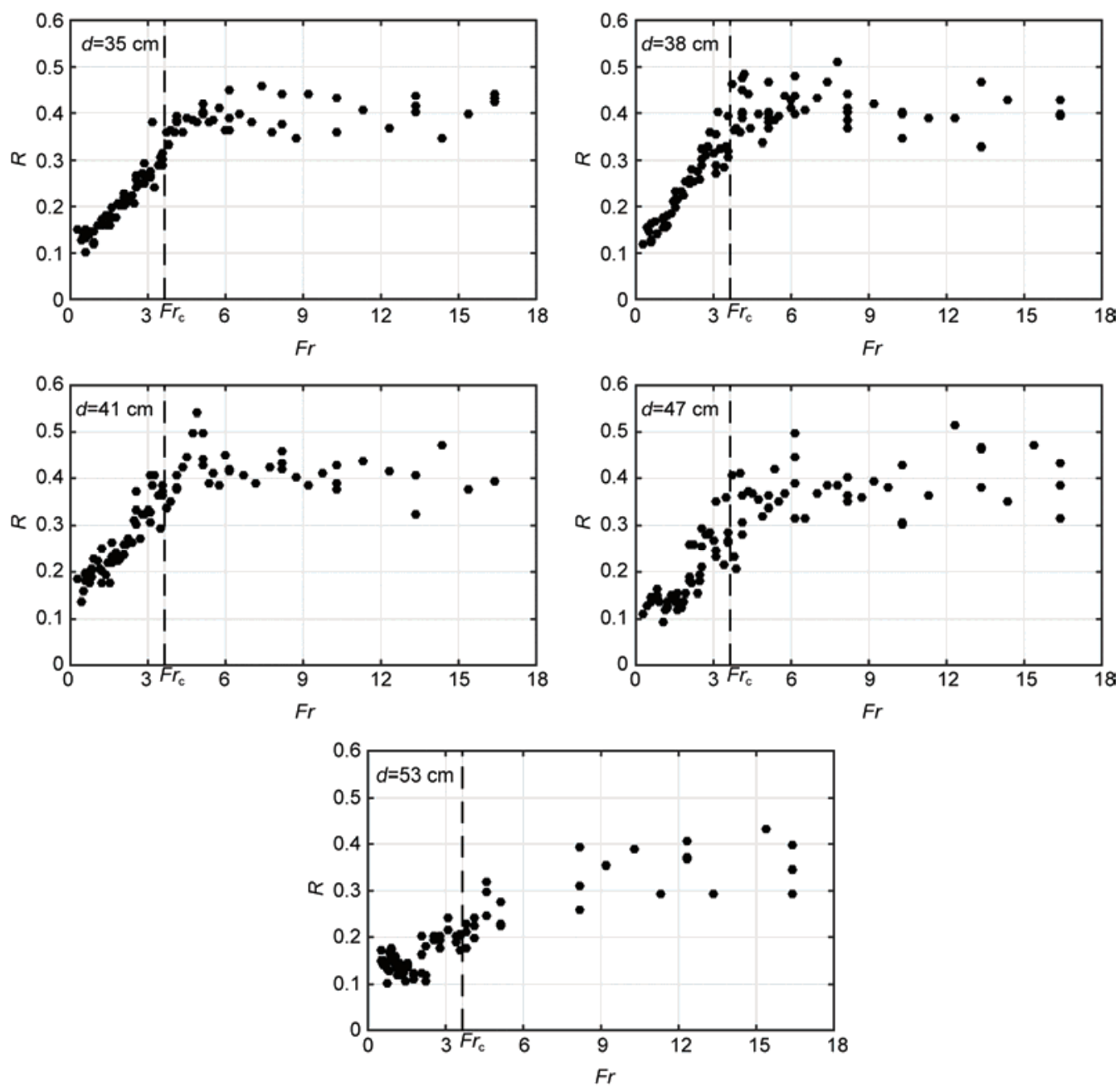

图 9 不同潜深下 $H_{\mathrm{m}} / D$ 随 $F r$ 的变化规律

Figure $9 H_{\mathrm{m}} / D$ versus $F r$ for five different submerged depths

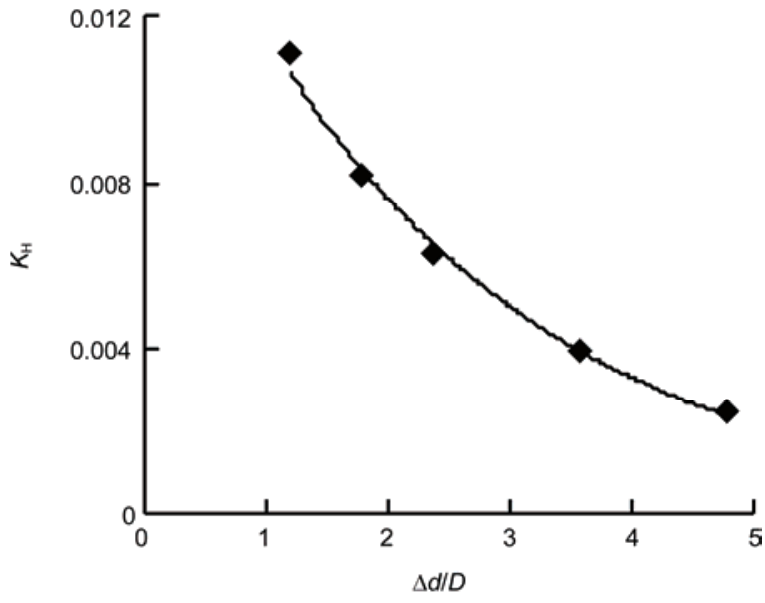

图 10 斜率 $k_{\mathrm{H}}$ 随 $\Delta d / D$ 的变化规律

Figure 10 Variation of $k_{\mathrm{H}}$ against dimensionless submerged depth $\Delta d / D$
当水下航行体下潜到海洋密度分层流体的密度跃层 下方某个深度后，其激发内波的幅值会很小，即水下 航行体可以利用其下潜深度等策略, 实现其对内波 尾迹的隐身之目的.

\section{3 内波时空形态特征}

本节讨论拖曳模型激发内波的时空形态特征, 包括体积效应和尾迹效应内波的波形和波系特征, 以及两类内波的对称性等. 为此, 首先简要陈述内波 的等相线理论. 设 $\varphi$ 为内波相位函数, 则根据驻相原 理, 一个移动点源激发内波的主要贡献来自使 $\partial \varphi /$ $\partial k=0$ 的点, 其中 $k$ 为波数. 记 $\omega_{m}=\omega_{m}(k)$ 为第 $m$ 个模态 的内波频率, 则相应模态的内波相速度 $c_{\mathrm{p} m}$ 和群速度 $c_{\mathrm{g} m}$ 为 


$$
c_{\mathrm{p} m}=\frac{\omega_{m}(k)}{k}, c_{\mathrm{g} m}=\frac{\mathrm{d} \omega_{m}(k)}{\mathrm{d} k} .
$$

由此可得, 第 $m$ 个模态内波的驻相点为

$$
x=\varphi \frac{U_{\mathrm{s}}\left(1-c_{\mathrm{p} m} c_{\mathrm{g} m} / U_{\mathrm{s}}^{2}\right)}{k\left(c_{\mathrm{p} m}-c_{\mathrm{g} m}\right)}, y=\varphi \frac{c_{\mathrm{g} m} \sqrt{1-c_{\mathrm{p} m}^{2} / U_{\mathrm{s}}^{2}}}{k\left(c_{\mathrm{p} m}-c_{\mathrm{g} m}\right)},
$$

其中, $U_{\mathrm{s}}$ 为内波激发源速度.

记 $\left(c_{\mathrm{p} m}\right)_{0}$ 为 $c_{\mathrm{p} m}$ 在 $k \rightarrow 0$ 时的极限值, 称为临界相速 度. 那么, 当 $U_{\mathrm{s}}>\left(c_{\mathrm{p} m}\right)_{0}$ 时, 该模态内波只有散波; 当 $U_{\mathrm{s}}<\left(c_{\mathrm{p} m}\right)_{0}$ 时, 该模态内波既有散波又有横波. 在本文 实验所获密度分层流体的浮力频率剖面下, 经计算 可得前两个模态的临界相速度分别为 19.4 和 $4.5 \mathrm{~cm} / \mathrm{s}$. 本文实验中拖曳速度的范围为 $5 \sim 160 \mathrm{~cm} / \mathrm{s}$, 该速度范 围均大于第二模态及更高阶模态内波的临界相速度. 因此, 在本文实验工况下, 二阶及更高阶模态内波只 有散波。

在图 11中, 给出了当潜深 $d=35 \mathrm{~cm}$ 时, 不同速度 下拖曳模型激发内波时空形态特征的实验结果. 其 中, 前 6 个速度为转捩前的情况, 后 4 个速度为转捩后
的情况. 此外, 图中横坐标为水槽横向坐标, 范围 为 $[-0.55,0.55]$ (单位为 $\mathrm{m}$ ), 纵坐标为时间, 范围 为 $[0,120]$ (单位为 $\mathrm{s}$ ). 由图可见, 在约 $40 \mathrm{~s}$ 之后, 由于 水槽侧壁的影响, 开始出现明显的内波反射现象.

首先分析转捩前的内波时空形态特征. 由图可 知, 当 $U=6 \mathrm{~cm} / \mathrm{s}$ 时, 可以明显地观察到两个模态的波 系, 其中外层为第二模态的波, 由于 $U>\left(c_{\mathrm{p} 2}\right)_{0}$, 因此 只有散波; 内层为第一模态的波，由于 $U<\left(c_{\mathrm{p} 1}\right)_{0}$, 因 此既有散波也有横波. 当 $U=12$ 和 $15 \mathrm{~cm} / \mathrm{s}$ 时, 第二模 态内波逐渐减弱，当 $U=15 \mathrm{~cm} / \mathrm{s}$ 时，第二模态内波几 乎不可见; 随着拖曳速度的增大，第一模态内波张角 逐渐增大，由于此时 $U$ 仍小于 $\left(c_{\mathrm{p} 1}\right)_{0}$ ，因此既有散波也 有横波. 当 $U=20,30$ 和 $35 \mathrm{~cm} / \mathrm{s}$ 时, 由于 $U>\left(c_{\mathrm{p} 1}\right)_{0}$, 因 此只有散波，而且散波张角随 $U$ 的增大变化较小.

在转捩后，拖曳模型的速度均大于 $\left(c_{\mathrm{p} 1}\right)_{0}$. 根据 驻相原理，此时内波将只有散波而没有横波.但由图 11(g) (j) 可见，转捩后的内波既有散波又有横波，而 且随着拖曳速度的增大，其空间形态特征变化较小. 进一步观察图11(c) (f), 可以发现：在转捩前内波时
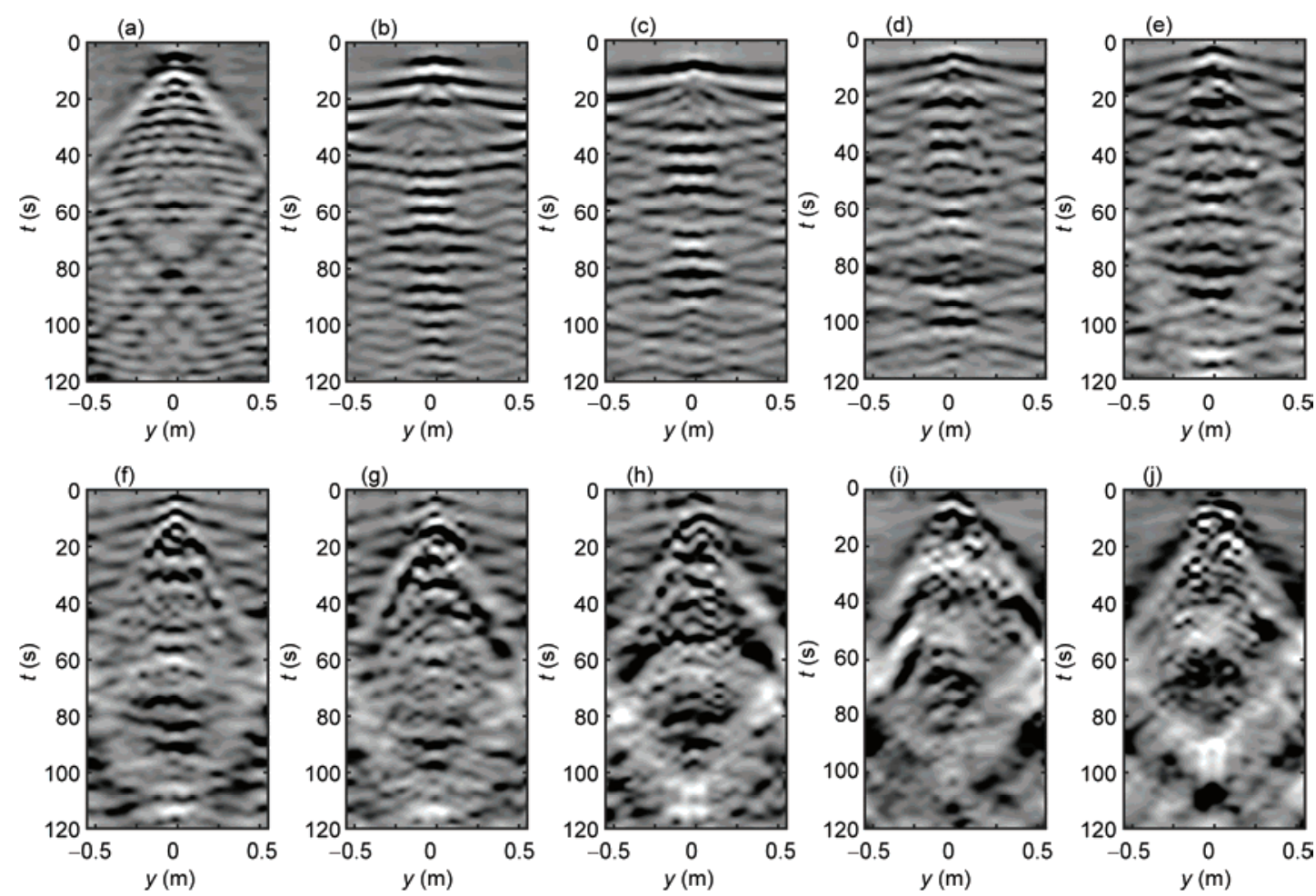

图 11 当潜深 $d=35 \mathrm{~cm}$ 时, 不同速度下拖曳模型激发内波的时空形态特征. (a) $6 \mathrm{~cm} / \mathrm{s}$; (b) $12 \mathrm{~cm} / \mathrm{s}$; (c) $15 \mathrm{~cm} / \mathrm{s}$; (d) $20 \mathrm{~cm} / \mathrm{s}$; (e) $30 \mathrm{~cm} / \mathrm{s}$; (f) 35 $\mathrm{cm} / \mathrm{s} ;$ (g) $40 \mathrm{~cm} / \mathrm{s}$; (h) $50 \mathrm{~cm} / \mathrm{s}$; (i) $80 \mathrm{~cm} / \mathrm{s}$; (j) $100 \mathrm{~cm} / \mathrm{s}$

Figure 11 Time-space wave patterns at different towing velocities when $d=35 \mathrm{~cm}$. (a) $6 \mathrm{~cm} / \mathrm{s}$; (b) $12 \mathrm{~cm} / \mathrm{s}$; (c) $15 \mathrm{~cm} / \mathrm{s}$; (d) $20 \mathrm{~cm} / \mathrm{s}$; (e) $30 \mathrm{~cm} / \mathrm{s}$; (f) $35 \mathrm{~cm} / \mathrm{s}$; (g) $40 \mathrm{~cm} / \mathrm{s}$; (h) $50 \mathrm{~cm} / \mathrm{s}$; (i) $80 \mathrm{~cm} / \mathrm{s}$; (j) $100 \mathrm{~cm} / \mathrm{s}$ 
空形态特征图中, 也存在这种既有横波又有散波且 空间形态特征变化较小的内波, 而且随着拖电速度 的增大, 其影响逐渐显著. 由此可见, 转㧖后尾迹效 应内波为主控内波, 其主要特征表现为: 既有横波又 有散波, 而且内波时空形态特征相似. 同时, 在转捩 前, 虽然体积效应内波为主控内波, 但在拖电速度较 大时，尾迹效应内波也是存在的.

由图还可发现, 尾迹效应内波关于水槽中纵剖 面是不对称的. 为进一步分析这种不对称现象, 在图 12 中, 给出了当潜深 $d=35 \mathrm{~cm}$ 时在 $P_{ \pm 3}$ 处拖曳模型产 生内波位移的时历特性. 由图可知, 当 $U=12$ 和 15 $\mathrm{cm} / \mathrm{s}$ 时, 在水槽侧壁尚未对内波产生反射之前(约 40 $\mathrm{s}$ 前), 两个对称位置处内波位移时历是一致的, 这表 明体积效应内波关于水槽中纵剖面是对称的. 当 $U=20,30$ 和 $35 \mathrm{~cm} / \mathrm{s}$ 时, 在约 $20 \mathrm{~s}$ 之前, 两个对称位置 处内波位移时历相位一致, 但随着速度增大幅值的 差异逐渐增大; 在约 $20 \mathrm{~s}$ 后, 相位和幅值均出现明显 的差异, 而且随着速度的增大, 这种差异愈加显著. 由此可见, 在转捩前随着拖曳速度的增大, 尾迹效应 内波的影响逐渐显著, 而且关于水槽中纵剖面是不 对称的.

在转捩后, 由图12(f) (h)可知, 从开始时刻两个 对称位置处内波位移时历的相位和幅值即有明显差 异, 这表明转捩后尾迹效应内波为主控内波, 而且关 于水槽中纵剖面明显是不对称的. 通过对其他潜深 下拖曳模型激发内波空间形态特征的分析, 所获结 果与潜深 $d=35 \mathrm{~cm}$ 的情况一致. 鉴于篇幅原因, 不再 细述.

\section{4 内波对称性分析}

设 $\Delta z=\Delta z(y, t)$ 为在点 $\left(0, y, z_{\mathrm{P}}\right)$ 处内波位移的时历. 熟知, 对任意一个实函数, 均可唯一地分解为一个偶 函数和一个奇函数之和. 据此, 将 $\Delta z$ 分解为关于 $y$ 的 偶函数和奇函数两个成分如下:

$\Delta z_{\mathrm{s}}=(\Delta z(y)+\Delta z(-y)) / 2, \Delta z_{\mathrm{a}}=(\Delta z(y)-\Delta z(-y)) / 2$. (9)

在上式中, $\Delta z_{\mathrm{s}}$ 是关于 $y$ 为偶函数, 该函数描述关 于水槽中纵剖面对称的内波成分; $\Delta z$ 是关于 $y$ 为奇函 数, 该函数描述关于水槽中纵剖面反对称的内波成 分. 为定量描述内波的反对称程度, 设 $\Delta z_{\mathrm{sj}}$ 和 $\Delta z_{\mathrm{a} j}$ 分别 为第 $j$ 个电导率探头所得内波位移时历的正对称和反 对称部分, $S_{\mathrm{s} j}$ 和 $S_{\mathrm{aj}}$ 分别为 $\Delta z_{\mathrm{sj}}$ 和 $\Delta z_{\mathrm{aj}}$ 的根均方差, 定义 内波反对称度 $R$ 如下:

$$
R=\frac{\sum_{j=-N}^{j=N(j \neq 0)} S_{a j}}{\sum_{j=-N}^{j=N(j \neq 0)}\left(S_{s j}+S_{a j}\right)} .
$$

由 2.2 节可知, 在 $-0.25 \mathrm{~m}<y<0.25 \mathrm{~m}$ 范围内, 内波 受水槽侧壁的影响较小. 为此, 对其 8 个电导率探头 $P_{ \pm 1}, P_{ \pm 2}, P_{ \pm 3}$ 和 $P_{ \pm 4}$ 所得内波位移时历进行分析, 即在 (10)式中, 取 $N=4$. 在图 13 中, 给出了不同潜深下反 对称度 $R$ 随Froude数 $F r$ 变化规律的实验结果. 由图可 知, 在转捩前, 随着 $F r$ 的增大, 反对称度 $R$ 也随着增 大, 当 $F r=F r_{\mathrm{c}}$ 时, 反对称度 $R$ 达到最大. 在转㧖后, 随 着 $F r$ 的增大, 反对称度 $R$ 近似在某个常数 $R_{\mathrm{c}}$ 附近波动, 并没有出现随着 $F r$ 的增大而增大的现象.

由图进一步可知, 在潜深 $d=35,38,41$ 和 $47 \mathrm{~cm}$ 的 情况中, 常数 $R_{\mathrm{c}}$ 近似为 0.4 . 而在潜深 $d=53 \mathrm{~cm}$ 的情况, 常数 $R_{\mathrm{c}}$ 有较为明显的减小, 近似为 0.35 , 即在某个潜 深后反对称度会出现明显减小的现象, 这意味着随 着潜深的增加, 水下航行体内波的反对称性会有一 定程度的减弱. 结果表明, 在转捩前, 水下航行体内 波中对称成分占优, 这与转捩前体积效应内波为主 控内波的结果一致. 在转捩后, 水下航行体内波的反 对称度一般不超过 0.5 , 其中对称性成分略为占优.

利用(9)式, 分离出内波位移时历的对称和反对 称部分, 进一步分析其对称和反对部分的时空形态 特征. 在图14中, 给出了转捩前拖曳模型激发内波对 称和反对部分的时空形态特征结果, 同时还给出了 相同速度下的内波等相线图. 其中, 等相线图中的虚 线为第二模态的波系，而实线为第一模态的波系.

由图可知, 内波对称部分的时空特征与相应速 度下的等相线理论预测结果一致, 而内波非对称部 分的时空特征与相应速度下的等相线理论预测结果 并不一致. 结合该速度下的内波反对称度的结果可 知, 转护前内波对称部分为主控内波, 而且其激发源 为物体的体积效应, 而非对称部分的激发源并不是 物体的体积效应, 而应该为尾迹效应.

由 2.1 节可知, 转捩后内波的相关速度并不等于 物体的运动速度, 即尾迹效应内波激发源的速度并 不与物体运动速度一致. 由文献 [5]可知, 水下拖曳 体尾迹中主控涡的Strouhal数 $S t=f D / U$ 的变化范围近 似为 $0.16 \sim 0.2$. 其中, $f$ 为主控浴的频率. 如果取 $S t=$ 0.16 , 那么可得主控浴的流向长度近似为 $L_{\mathrm{eddy}}=U / f \approx$ $6.25 D$, 激励频率近似为 $f=U_{\text {eddy }} / L_{\text {eddy }} \approx 0.16 U_{\text {eddy }} / D$, 其中 $U_{\text {eddy }}(<U)$ 为主控浴移动速度. 由 2.1 节的分析可 

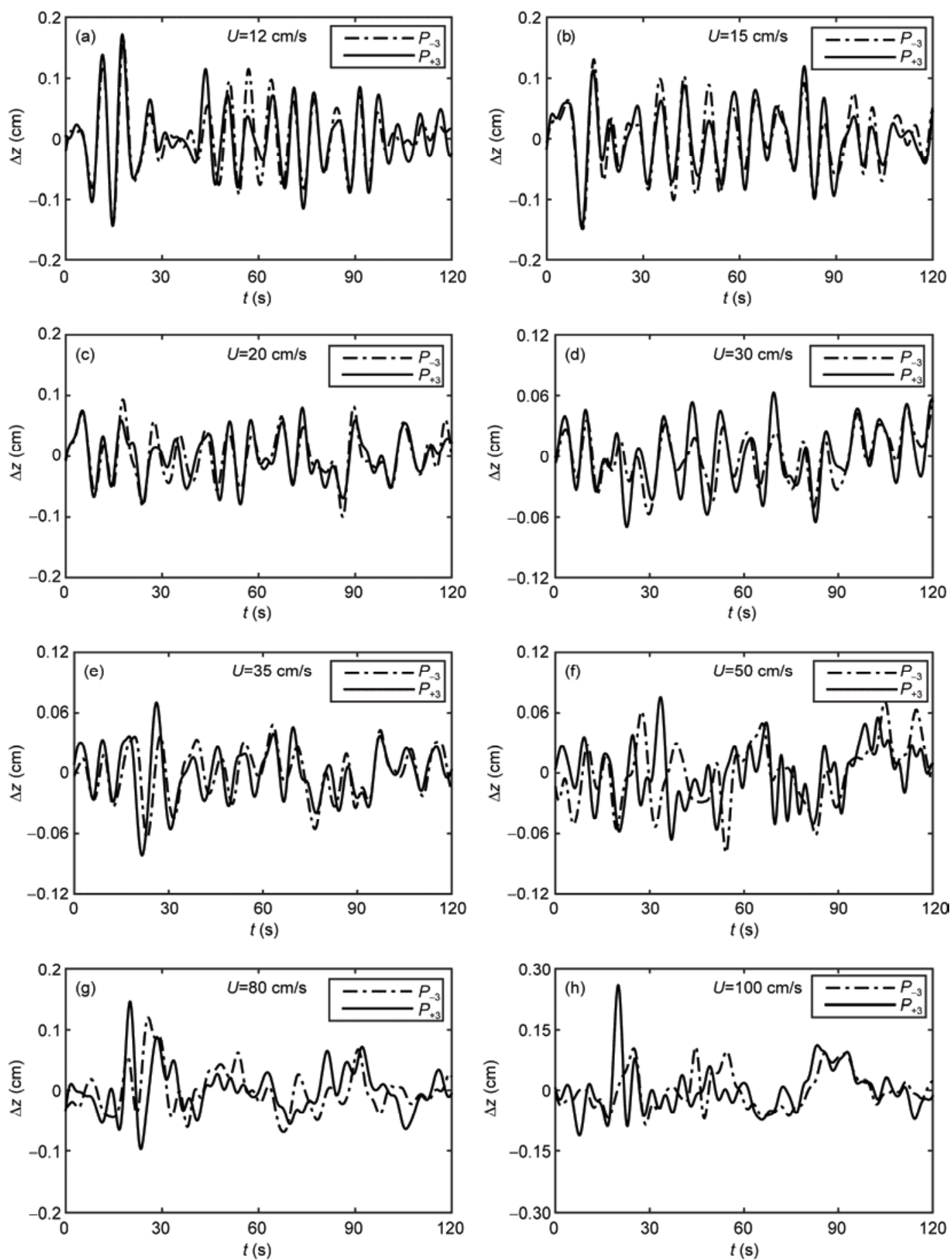

图 12 当潜深 $d=35 \mathrm{~cm}$ 时, 不同速度下拖曳模型激发内波位移时历特性. (a) $12 \mathrm{~cm} / \mathrm{s}$; (b) $15 \mathrm{~cm} / \mathrm{s}$; (c) $20 \mathrm{~cm} / \mathrm{s}$; (d) $30 \mathrm{~cm} / \mathrm{s}$; (e) $35 \mathrm{~cm} / \mathrm{s}$; (f) $50 \mathrm{~cm} / \mathrm{s}$; (g) $80 \mathrm{~cm} / \mathrm{s}$; (h) $100 \mathrm{~cm} / \mathrm{s}$

Figure 12 Time series of $\Delta z$ at different towing speeds when $d=35 \mathrm{~cm}$. (a) $12 \mathrm{~cm} / \mathrm{s}$; (b) $15 \mathrm{~cm} / \mathrm{s}$; (c) $20 \mathrm{~cm} / \mathrm{s}$; (d) $30 \mathrm{~cm} / \mathrm{s}$; (e) $35 \mathrm{~cm} / \mathrm{s}$; (f) $50 \mathrm{~cm} / \mathrm{s}$; (g) $80 \mathrm{~cm} / \mathrm{s}$; (h) $100 \mathrm{~cm} / \mathrm{s}$

知, 转捩后内波相关速度Froude数 $F r_{\mathrm{iw}}$ 在 $0.7 \sim 1.4$ 之间 的一个条带内变化, 条带均值约为 1.0 . 为此, 将 $F r_{\mathrm{iw}}$ 近似取为 1.0 , 那么由(1)式可知, 转捩后内波相关速
度近似为 $U_{\mathrm{iw}}=F r_{\mathrm{iw}} D N_{\text {max }} \approx D N_{\text {max }}$. 将主控涡移动速度 取为内波相关速度, 即 $U_{\text {eddy }}=U_{\text {iw }}$, 那么可得 $f \approx N_{\text {max }} / 2 \pi$, 正好近似为密度分层流体最大浮力频率(圆频率). 

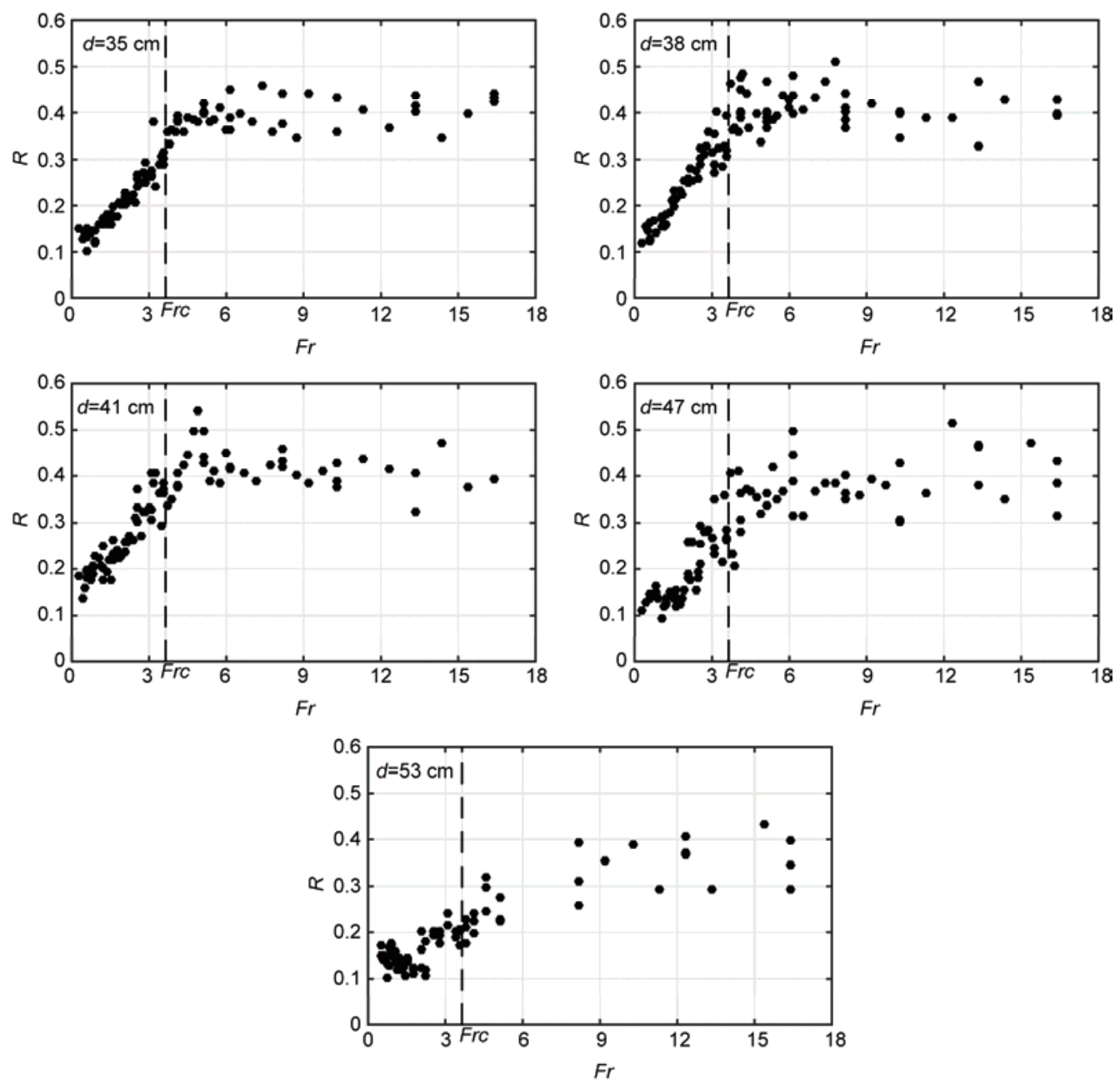

图 13 不同潜深下, 反对称度 $R$ 随Froude数 $F r$ 的变化规律

Figure 13 Degree of anti-symmetry $R$ versus $F r$ for different submerged depths

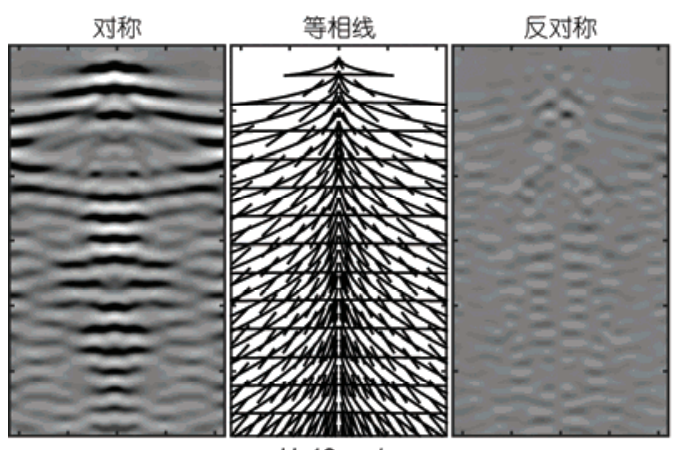

$U=12 \mathrm{~cm} / \mathrm{s}$

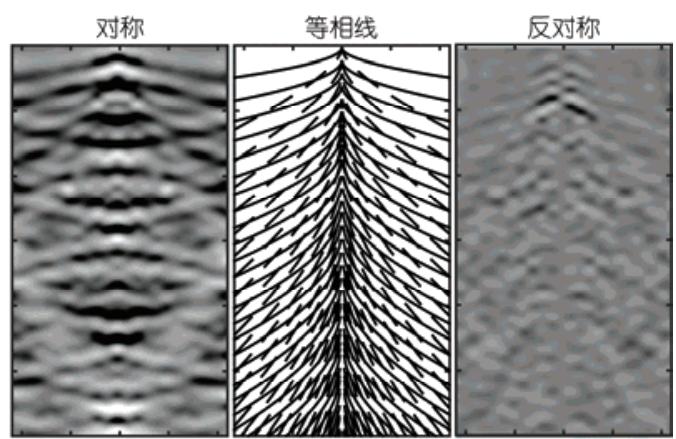

$U=30 \mathrm{~cm} / \mathrm{s}$

图 14 当潜深 $d=35 \mathrm{~cm}$ 时, 转捩前内波对称和反对称部分的时空形态特征

Figure 14 Symmetric and anti-symmetric components of time-space wave patterns before transition when $d=35 \mathrm{~cm}$

意味着转捩后相关速度Froude数为 $F r_{\mathrm{iw}} \approx 1.0$ 的内波, 将是尾迹效应内波中最为显著的一类内波.

下面通过分析拖曳模型激发内波对称和反对称
部分的时空形态特征, 来说明上述陈述的合理性. 在 本文实验工况下，由 $F r_{\mathrm{iw}} \approx 1.0$ 可得，相应的内波相关速 度为 $U_{\mathrm{iw}} \approx 9.6 \mathrm{~cm} / \mathrm{s}$. 在图 15 中, 给出了转捩后拖曳模型 


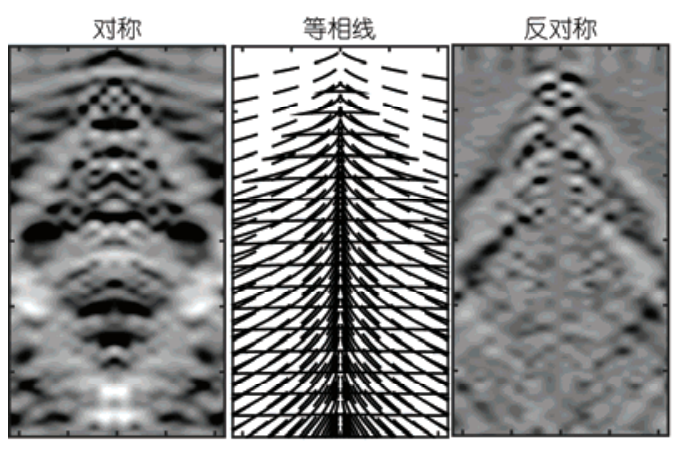

$U=50 \mathrm{~cm} / \mathrm{s}$

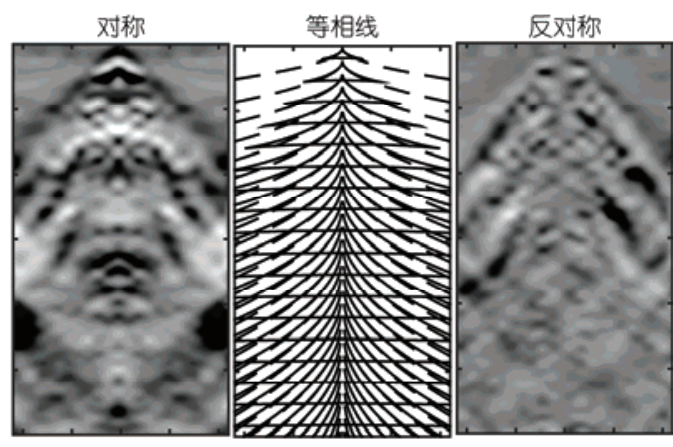

$U=80 \mathrm{~cm} / \mathrm{s}$

图 15 当潜深 $d=35 \mathrm{~cm}$ 时, 转捩后内波对称和反对称部分的时空形态特征

Figure 15 Symmetric and anti-symmetric components of time-space wave patterns after transition when $d=35 \mathrm{~cm}$

激发内波对称和反对称部分的时空形态特征结果, 同时还给出了相关的内波等相线图. 其中, 实线为 $U_{\mathrm{iw}} \approx 9.6 \mathrm{~cm} / \mathrm{s}$ 时的等相线, 而虚线为 $U_{\mathrm{s}}=U$ (模型拖曳 速度)时的等相线.

由图可知, 当 $U=50 \mathrm{~cm} / \mathrm{s}$ 时, 内波对称部分外层 时空形态特征与 $U_{\mathrm{s}}=U$ 时的等相线一致, 这表明虽然 转㧖后尾迹效应内波为主控内波, 但仍存在体积效 应内波的成分; 内波对称部分的内层时空形态特征 与 $U_{\mathrm{iw}} \approx 9.6 \mathrm{~cm} / \mathrm{s}$ 时的等相线近似一致, 而且反对称部 分的时空形态特征与 $U_{\mathrm{iw}} \approx 9.6 \mathrm{~cm} / \mathrm{s}$ 时的等相线也近 似一致. 当 $U=80 \mathrm{~cm} / \mathrm{s}$ 时, 对称部分中的体积效应内 波已不可见, 这表明转捩后随着拖曳速度的增大体 积效应内波逐渐减弱, 直至消失; 此时, 内波对称部 分的时空形态特征与 $U_{\mathrm{iw}} \approx 9.6 \mathrm{~cm} / \mathrm{s}$ 时的等相线近似 一致, 而且反对称部分的时空形态特征与 $U_{\mathrm{iw}} \approx 9.6$ $\mathrm{cm} / \mathrm{s}$ 时的等相线也近似一致.

对尾迹内波的水平张角 $\theta_{c}$, Voisin ${ }^{[29]}$ 给出了一个 理论估算公式如下:

$$
\theta_{c}=\frac{2}{3^{3 / 2} \gamma}=\frac{0.385}{\gamma}, \gamma=2 \pi F r_{\text {iw }} S t .
$$

根据本文实验结果, 将尾迹内波相关速度 Froude数 $F r_{\text {iw }}$ 取为 1.0 , 而尾迹涡 Strouhal数 $S t$ 取为 0.16 , 则由 (11)式可得, $\theta_{c}=21.9^{\circ}$. 将图 15中灰度图的 时间纵坐标按 $x=U_{\mathrm{s}} t$ 换算为空间坐标, 可得转捩后拖 曳模型激发内波对称和反对称部分的空间形态特征 如图 16所示. 其中, 纵坐标和横坐标的单位均为 $\mathrm{cm}$, 图中实线为尾迹内波的近似外包络线, 反映其水平 张角. 由图可知, 在转捩后, 在不同拖曳速度下, 尾 迹内波对称和反对称部分的水平张角均近似为 $\theta_{c}=20.6^{\circ}$, 这与 $\mathrm{Voisin}^{[29]}$ 的理论预测结果一致. 由此
可见, 将转捩后尾迹效应内波的相关速度Froude数 $F r_{\text {iw }}$ 近似取为 1.0 是合理的.

目前关于水下航行体激发内波的理论模型及相 关的实验研究中, 都先验地假定一个轴对称物体激 发的内波关于物体中纵剖面是对称的. 本文的研究 表明，对水下航行体激发的尾迹内波，这种先验假定 是错误的. 由 2.1 节的分析可知, 在实际海洋中水下 航行体激发的内波主要以尾迹内波为主控内波. 因 此, 建立能够完整描述尾迹效应内波中对称和反对 称两类成分特性的理论模型, 不仅有重要的理论意 义, 而且具有重要的实际应用价值.

等效质量源理论模型是目前预测水下航行体激 发内波特性的主要方法之一. 但在该方法中, 采用一 个轴对称的等效质量源来模拟内波的激发源, 因此 该方法不能模拟反对称的内波. 有鉴于此, 本文提出 等效质量源的一种新的构造思路如下:

构造一对关于 $y$ 轴对称的等效质量源，用来模 拟尾迹内波中的对称成分; 同时，构造一对关于 $y$ 轴 反对称的等效质量源，用来模拟尾迹内波中的反对 称成分. 根据前面的分析可知，尾迹内波的对称和反 对称成分, 主要来自于相关速度Froude数 $F r_{\mathrm{iw}}$ 近似为 1.0 内波的贡献. 因此, 两类对称和反对称等效质量 源的移动速度均可近似取为 $U_{\mathrm{s}} \approx D N_{\max }$. 有关该思路 的可行性等问题, 目前正在研究之中.

\section{3 结论}

在中间层为强跃层的密度分层流体中, 利用沿 水槽中纵剖面对称布置的电导率探头阵列测量技术, 对长径比为 7.7 的细长回转体在 5 个不同潜深下激发 内波特性开展了系列实验. 采用相关性分析方法, 结 

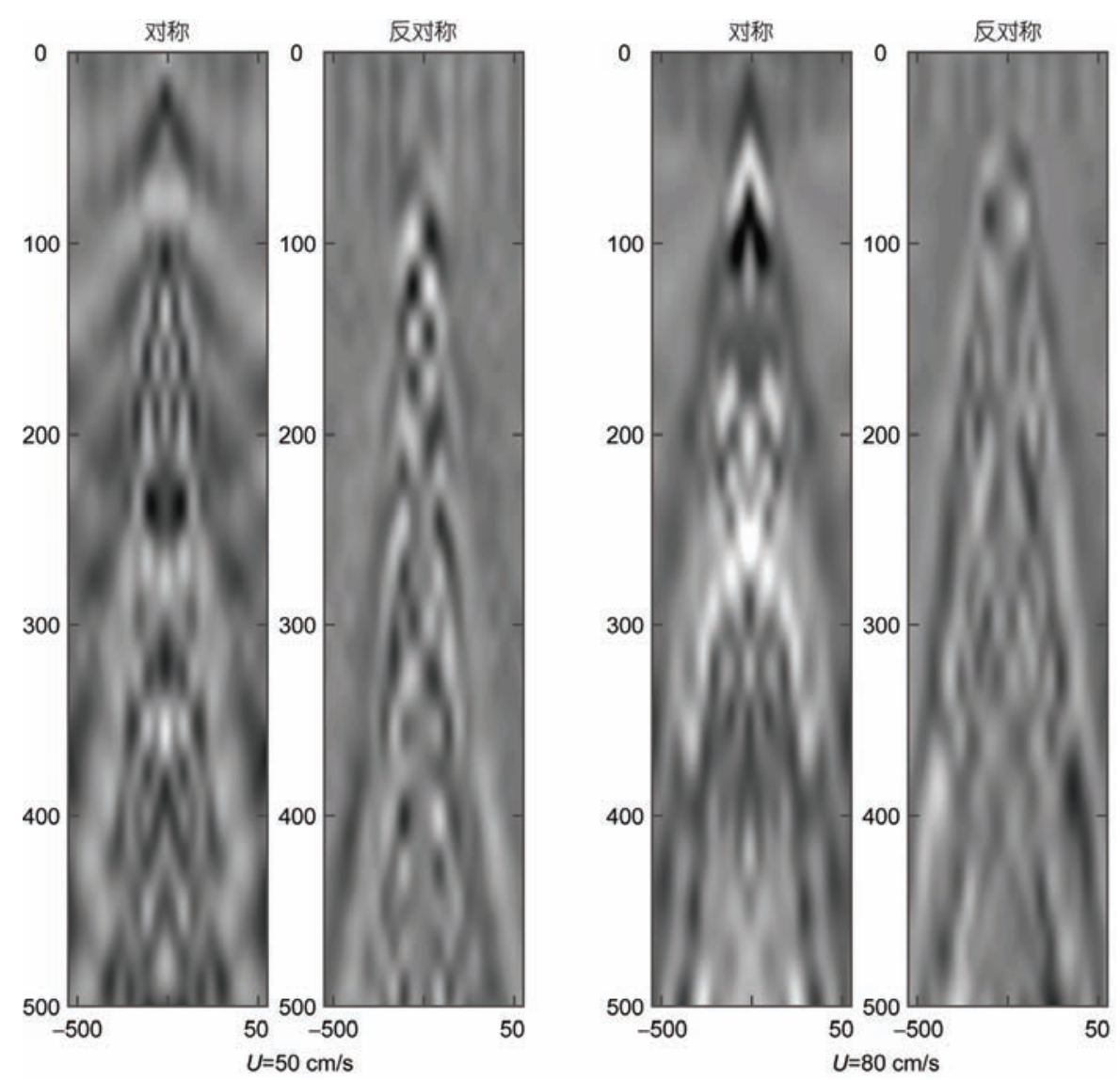

图 16 转捩后内波对称和反对称部分的空间形态特征

Figure 16 Symmetric and anti-symmetric components of spacial wave patterns after transition

合根均方差及奇偶函数分解等手段, 分析了体积效 应和尾迹效应内波的转捩特性、位移场特性、时空形 态特征和对称性等, 获得了如下结果:

对不同潜深, 均存在一个相同的临界Froude数 $F r_{\mathrm{c}}$, 它与长径比Froude数 $\lambda$ 近似满足线性关系 $F r_{\mathrm{c}}=$ $0.2391 \lambda+1.7679$; 当 $F r<F r_{\mathrm{c}}$ 时, 内波相关速度与物体 运动速度一致, 体积效应内波为主控内波; 当 $F r>F r_{\mathrm{c}}$ 时, 内波相关速度小于物体运动速度, 尾迹效应内波 为主控内波, 而且内波相关速度 Froude数 $F r_{\text {iw }}$ 在 0.7 1.4之间的一个条带内变化.

在转捩前, 内波位移时历根均方差近似与高斯 分布一致, 而且在水槽中纵剖面处达到最大值. 转捩 后, 内波位移时历根均方差关于 $y$ 并不是对称的, 而 且并不一定在水槽中纵剖面处达到其最大值. 这表 明, 体积效应内波关于水槽中纵剖面是对称的, 而尾 迹效应内波关于水槽中纵剖面是不对称的.

在不同潜深下，转捩前均存在一个近似相同的 峰值Froude数 $F r_{\mathrm{p}}=1.33$, 当 $F r<F r_{\mathrm{p}}$ 时, 无量纲内波最
大峰-峰幅值随 $F r$ 增大而增大; 当 $F r_{\mathrm{p}}<F r<F r_{\mathrm{c}}$ 时, 无 量纲内波最大峰-峰幅值随 $F r$ 增大而减小. 在转捩后, 无量纲内波最大峰-峰幅值随 $F r$ 增大而近似线性增 大，其线性增大的斜率与潜深之间近似满足指数衰 减关系 $k_{\mathrm{H}}=0.0176 \mathrm{e}^{-0.418 \Delta d / D}$.

在不同潜深下，转捩前内波反对称度均随 $F r$ 增 大而增大, 而且当 $F r=F r_{\mathrm{c}}$ 时达到最大, 这表明虽然转 捩前对称部分的体积效应内波为主控内波，但非对 称部分的尾迹效应内波随 $F r$ 的增大其影响逐渐增大 在转捩后, 内波反对称度近似在某个小于 0.5 的常数 附近波动，但在某潜深后反对称度出现明显减小的 现象，这表明尾迹效应内波中对称部分略为占优．同 时, 转捩后相关速度Froude数为 $F r_{\mathrm{iw}} \approx 1.0$ 的内波, 是 尾迹效应内波中最为显著的一类内波，而且在不同 潜深和不同拖曳速度下，尾迹效应内波中对称和反 对称部分的时空形态特征相似，而且其水平张角均 近似为 $20.6^{\circ}$.

通过本文的系列模型实验和分析，进一步深化 
了水下运动物体激发内波转捩特性、波高特性、时空 形态特征及其对称性等问题的认识, 并为后续建立
能够完整描述尾迹效应内波中对称和反对称两类内 波特性的理论模型，提供了一定的理论依据.

\section{参考文献}

1 Xu Z T. Dynamics of ocean internal waves (in Chinese). Beijing: Science Press, 1999 [徐肇廷. 海洋内波动力学. 北京: 科学出版社, 1999]

2 Wei G, Dai S Q. Advances in internal waves due to moving body in stratified fluid systems (in Chinese). Adv Mech, 2006, 36: 111-124 [魏岗, 戴世强. 分层流体中运动源生成的内波研究进展. 力学进展, 2006, 36: 111-124]

3 Brandt A, Rottier J R. The internal wavefield generated by a towed sphere at low Froude number. J Fluid Mech, 2015, 769: 103-129

4 Spedding G R. Wake signature detection. Annu Rev Fluid Mech, 2014, 46: 273-302

5 Chomaz J M, Bonneton P, Hopfinger E J. The structure of the near wake of a sphere moving horizontally in a stratified fluid. J Fluid Mech, 1993, 254: 1-21

6 Brandt A, Schemm C E. Small-scale structure in the near field of a stratified wake. In: Proceedings of 7th International Symposium on Stratified Flows. Rome, 2011

7 Bonnier M, Eiff O. Experimental investigation of the collapse of a turbulent wake in a stably stratified fluid. Phys Fluids, 2002, 14: 791-801

8 Meunier P, Diamessis P J, Spedding G R. Self-preservation in stratified momentum wakes. Phys Fluids, 2006, 18: 106601

9 Chen K, You Y, Noblesse F. Experimental study of quasi-2D dipolar vortex streets generated by a moving momentum source in a stratified fluid. Phys Fluids, 2016, 28: 075105

10 Chernyshenko S I, Castro I P. High-Reynolds-number weakly stratified flow past an obstacle. J Fluid Mech, 1996, 317: 155-178

11 Plougonven R, Zeitlin V. Internal gravity wave emission from a pancake vortex: An example of wave-vortex interaction in strongly stratified flows. Phys Fluids, 2002, 14: 1259-1268

12 Sutherland B R, Flynn M R, Dohan K. Internal wave excitation from a collapsing mixed region. Deep Sea Res Part II, 2004, 51: 2889-2904

13 Hopfinger E J, Flor J B, Chomaz J M, et al. Internal waves generated by a moving sphere and its wake in a stratified fluid. Exp Fluids, 1991, 11: 255-261

14 Lin Q, Boyer D L, Fernando H J S. Internal waves generated by the turbulent wake of a sphere. Exp Fluids, 1993, 15: 147-154

15 Bonneton P, Chomaz J M, Hopfinger E J. Internal waves produced by the turbulent wake of a sphere moving horizontally in a stratified fluid. J Fluid Mech, 1993, 254: 23-40

16 Robey H F. The generation of internal waves by a towed sphere and its wake in a thermocline. Phys Fluids, 1997, 9: 3353-3367

17 Wei G, Zhao X Q, Su X B, et al. Experimental study on time series structures of the wake in a linearly stratified fluid (in Chinese). Sci Sin Ser G-Phys Mech Astron, 2009, 39: 1338-1347 [魏岗, 赵先奇, 苏晓冰, 等. 分层流体中尾迹时间序列结构的实验研究. 中国科学 G 辑: 物理 学 力学 天文学, 2009, 39: 1338-1347]

18 Zhao X Q, You Y X, Chen K, et al. Experimental study on the generation of internal waves by a slender body in stratified fluid (in Chinese). J Shanghai Jiao Tong Univ, 2009, 43: 1298-1301 [赵先奇, 尤云祥, 陈科, 等. 分层流体中细长体生成内波的试验研究. 上海 交通大学学报, 2009, 43: 1298-1301]

19 Wang J, You Y X, Hu T Q, et al. The characteristics of internal waves generated by a revolution body in a stratified fluid with a pycnocline (in Chinese). Acta Phys Sin, 2012, 61: 074701 [王进，尤云祥，胡天群，等. 具有密度跃层分层流体中回转体激发内波特性实 验. 物理学报, 2012, 61: 074701]

20 Wang J, You Y X, Hu T Q, et al. The characteristics of internal waves excited by towed bodies with different aspect ratios in a stratified fluid (in Chinese). Chin Sci Bull, 2012, 57: 606-617 [王进，尤云祥，胡天群，等. 密度分层流体中不同长径比拖曳潜体激发内波特 性实验. 科学通报, 2012, 57: 606-617]

21 Keller J B, Munk W H. Internal wave wakes of a body moving in a stratified fluid. Phys Fluids, 1970, 13: 1425-1431

22 Broutman D, Rottman J W, Eckermann S D. Ray methods for internal waves in the atmosphere and ocean. Annu Rev Fluid Mech, 2004, 36: $233-253$

23 Scase M M, Dalziel S B. Internal wave fields generated by a translating body in a stratified fluid: an experimental comparison. J Fluid Mech, 2006, 564: 305-331

24 Voisin B. Lee waves from a sphere in a stratified flow. J Fluid Mech, 2007, 574: 273-315

25 You Y X, Zhao X Q, Chen K, et al. An equivalent mass source method for internal waves generated by a body moving in a stratified fluid of finite depth (in Chinese). Acta Phys Sin, 2009, 58: 6750-6760 [尤云祥, 赵先奇, 陈科, 等. 有限深密度分层流体中运动物体生成 内波的一种等效质量源方法. 物理学报, 2009, 58: 6750-6760]

26 Lin Q, Boyer D L, Fernando H J S. Turbulent wakes of linearly stratified flow past a sphere. Phys Fluids, 1992, 4: 1687-1696

27 Xie J, Chen Z, Xu J, et al. Effect of vertical stratification on characteristics and energy of nonlinear internal solitary waves from a numerical model. Commun Nonlinear Sci Numer Simulat, 2014, 19: 3539-3555

28 Cai S, Xie J, Xu J, et al. Monthly variation of some parameters about internal solitary waves in the South China Sea. Deep Sea Res Part I, 2014, 84: 73-85

29 Voisin B. Internal wave generation by turbulent wakes. Mixing Geophys Flows, 1995. 291-301 


\title{
Experiments on internal waves generated by a towed revolution body with different depths in a stratified fluid
}

\author{
WANG HongWei ${ }^{1,2}$, CHEN Ke ${ }^{1,2^{*}}$, YOU YunXiang ${ }^{1,2} \&$ ZHANG XinShu ${ }^{1,2}$ \\ ${ }^{1}$ State Key Laboratory of Ocean Engineering, Shanghai Jiao Tong University, Shanghai 200240, China; \\ ${ }^{2}$ Collaborative Innovation Center for Advanced Ship and Deep-Sea Exploration, Shanghai 200240, China \\ * Corresponding author, E-mail: raulphan@sjtu.edu.cn
}

Internal waves generated by submerged bodies such as towed spheres, towed cylinders or self-propelled models in stratified fluids are of great interests in oceanic hydrodynamics. Several classifications of internal waves were proposed based on experimental observations, including the Lee waves, the random waves and the gravitational collapsed waves. A more general classification is proposed in recent studies, consisting only two types. The first type is the body-generated internal wave which is stationary to the towed sphere and the second is the wake-generated internal wave which is non-stationary to the towed sphere. According to Robey's work, experiments in this paper were performed for the time-space characteristics of internal waves generated by a horizontally towed revolution slender body with the aspect ratio $\lambda=7.7$, located at five different submerged depths below the strong pycnocline of a stratified fluid. The Reynolds number range is from $2500<R e=U D / v<80000$ and the Froude number range from $0.3<F r=U / N_{\max } D<16.7$. Based on the real-time measurements of conductivity probe arrays which were arranged symmetrically in transverse section of the stratified fluid tank, and by means of the cross correlation analysis of the density perturbation between the two probe arrays arranged at different along-track positions, it is shown that the transition between the body-generated internal wave and the wake-generated internal wave is related to a critical Froude number $F r_{\mathrm{c}}=3.64$ which is independent of submerged depths of the revolution slender body. For $F r<F r_{\mathrm{c}}$, the internal waves are dominated by body-forced effect, and their correlation velocities are equal to the towing speeds of the revolution slender body, as well as the degree of anti-symmetry for such internal waves becomes larger with the increasing $F r$. Moreover, the dimensionless maximum peak-peak amplitudes for such internal waves dominated by body-forced effect firstly increase with the growth of $\mathrm{Fr}$ until $\mathrm{Fr}$ reaches a certain value of approximately 1.33 regardless of submerged depths, and then begin to decrease. For $F r>F r_{c}$, the internal waves are dominated by wake-forced effect, and their correlation velocities are noticeably lower than the towing speeds of the revolution slender body, as well as the Froude numbers with respect to the correlation velocities for such internal waves vary within the range from 0.7 to 1.4. Results further show that the degree of anti-symmetry for such wake-generated internal waves fluctuates around a constant smaller than 0.5 , indicating that the symmetric component is slight stronger than the anti-symmetric component in the wake-generated waves, as well as the opening angles of the wave patterns in the symmetric and anti-symmetric components remain at relatively constant value of approximately $20.6^{\circ}$ regardless of submerged depths and towed speeds of the revolution slender body. Moreover, the dimensionless maximum peak-peak amplitudes for such internal waves dominated by wake-forced effect linearly increase with $\mathrm{Fr}$ for different submerged depths, the slopes of such linear relations decrease with submerged depths and follow an exponential law. In particular, the present work demonstrates that it is unreasonable to assume that the wakegenerated internal waves for an axis-symmetric revolution body are symmetric about the longitudinal section in center plane of the body.

stratified fluid, axis-symmetric revolution body, body-generated internal waves, wake-generated internal waves, degree of anti-symmetry

doi: 10.1360/N972016-00864 